\title{
Heterogeneous systems in $d$ dimensions: lower spectrum
}

\author{
Paolo Amore
}

Facultad de Ciencias, CUICBAS, Universidad de Colima, Bernal Díaz del Castillo 340, Colima, Colima, Mexico

\begin{abstract}
We show that the properties of the lower part of the spectrum of the Helmholtz equation for an heterogeneous system in a finite region in $d$ dimensions, where the solutions to the homogeneous problems are known, can be systematically approximated by means of iterative methods. These methods only require the specification of an arbitrary ansatz and necessarily converge to the desired solution, regardless of the strength of the inhomogeneities, provided that the ansatz has a finite overlap with it. Different boundary conditions at the borders of the domain can be assumed. Applications in one and two dimensions are used to illustrate the methods.
\end{abstract}

Keywords: Helmholtz equation; inhomogeneous drum;

\section{Introduction}

In this paper we study the properties of heterogeneous systems in one or more dimensions obeying the Helmholtz equation

$$
\left(-\Delta_{d}\right) \Psi_{n}\left(x_{1}, \ldots, x_{d}\right)=E_{n} \Sigma\left(x_{1}, \ldots, x_{d}\right) \Psi_{n}\left(x_{1}, \ldots, x_{d}\right)
$$

where $\Delta_{d}$ is the Laplacian in a region $\Omega_{d}$ in $d$ dimensions, $\Sigma\left(x_{1}, \ldots, x_{d}\right)>0$ is the density and $\Psi_{n}$ and $E_{n}$ are the eigenfunctions and eigenvalues of the equation respectively. The eigenfunctions of the equation satisfy appropriate boundary conditions on $\partial \Omega_{d}$.

Heterogeneous systems are naturally present in nature, where the density of the medium has typically random or granular properties, but they can also be artificially created in the laboratory, generally with periodic properties. In particular the study of composite materials, built of alternating homogeneous layers of materials with different properties (density, conductivity, refraction index, etc.), is nowadays gaining importance in different areas of Physics and Engineering.

Examples of these systems are numerous and a full account of the bibliography on the subject lies outside the scope of this paper but, to give an idea, we can mention composite membranes [1, 3, 2, 4, 5], periodic multi-layered acoustic waveguides [6], elastic properties in layered media [7, 8, 9, 10, 11, 12], acoustic waves in strongly heterogeneous porous structures [13] or photonic crystals [14]. Particularly interesting, both from a physical and mathematical viewpoint, is the case of periodically layered composite materials, where the typical scale of the micro-structure

Email address: paolo.amore@gmail.com (Paolo Amore) Preprint submitted to Elsevier 
(i.e. the period of the layers) is much smaller than the macroscopic scale of the object. The understanding of the macroscopic properties of such systems in terms of their microscopic features is the fundamental goal of the "homogenization method" (see ref. [15] and references therein).

Our present analysis is restricted to the class of problems described by Eq. (1), which is a subset of the general problems listed above, although the basic ideas of the method might be used in more general situations. Our goal is to devise a method which allows one to obtain arbitrarily precise approximations to the eigenfunctions and eigenvalues corresponding to the lower part of the spectrum regardless of the specific details of the problem under consideration, such as the geometrical properties of $\Omega_{d}$ 1, the boundary conditions and, even more crucially, the density.

Here we describe an iterative method which progressively builds one of the eigenfunctions of the equation above corresponding to the lower states, starting from an almost arbitrary ansatz.

The salient features of our approach are

- The method applies to $d$-dimensional regions where an orthonormal basis of the Laplacian is known, with the exception of $d=2$ where this limitation can be circumvented using a conformal mapping which transforms the original simply connected region into a region where a basis is known;

- Different boundary conditions on $\partial \Omega_{d}$ can be enforced;

- The convergence of the method is not limited to cases where mild inhomogeneities are present: in other words, the method is truly non-perturbative;

- The rate of convergence can be increased using a judicious choice of the initial ansatz, which can also include variational parameters;

- If the density depends on some perturbative parameter $\lambda$, and it reduces to the homogeneous case for $\lambda \rightarrow 0$, the iterative method allows one to extract the perturbative expansion for the eigenfunction and eigenvalue of the targeted state to any order, with an appropriate number of iterations.

All these aspects are illustrated in the paper considering examples in one and two dimensions. In particular, we examine the case of a one dimensional problem with rapidly oscillating density and different boundary conditions (we consider the cases of Dirichlet, mixed Dirichlet-Neumann, Neumann and periodic bc) and we obtain the exact asymptotic behavior of the lower eigenvalues of Eq. (1) when the size of the micro-structure goes to zero. For the case of Dirichlet bc the result contains results previously published as a special case [16, 17].

The paper is organized as follows: in Section 2 we describe the method and prove theorems which apply both to the case where the spectrum of the problem does not contain a zero mode and to the case where a zero mode is present; in Section 3 we consider few applications of the method in one and two dimensions, in Section [4 we draw our conclusion and discussion possible extensions of the present work.

\section{The method}

In this section we describe different approaches which can be used to obtain approximations to the normal modes of the Helmholtz equation for a $d$ dimensional region with a variable density. The techniques that we will discuss do not require that the inhomogeneities are small and

${ }^{1}$ For $d \neq 2$ the method requires that the solutions to the homogeneous problem are known. 
the convergence towards the targeted state is always granted. The choice of a suitable ansatz containing variational parameters may increase the convergence.

We first discuss the calculation of the fundamental mode and state the following theorem:

Theorem 1. (Spectrum without zero mode) Consider a d dimensional region of space, $\Omega_{d}$, and a density $\Sigma\left(x_{1}, \ldots, x_{d}\right)>0$ which varies over $\Omega_{d}$; let $\Xi^{(0)}\left(x_{1}, \ldots, x_{d}\right)$ be an arbitrary function defined on $\Omega_{d}$, which has an overlap with the lowest mode of the Helmholtz equation (1) where $n$ is the set of numbers which specify an eigenfunction.

We assume that the spectrum of Eq. (I) does not contain a zero mode (i.e. a mode with vanishing eigenvalue), and therefore that its eigenfunctions obey specific boundary conditions on $\partial \Omega_{d}$ (in one dimension, for instance, only Dirichlet boundary conditions at both ends, or mixed Dirichlet and Neumann boundary conditions at each end are allowed).

Let $G\left(x_{1}, \ldots, x_{d} ; y_{1}, \ldots, y_{d}\right)$ be the Green's function of the homogeneous problem on $\Omega_{d}$ obeying the same boundary conditions of $\Psi$. We call $\Psi_{0}\left(x_{1}, \ldots, x_{d}\right)$ the eigenfunction of the fundamental mode.

Then, for $p \rightarrow \infty$, the sequence of functions

$$
\begin{aligned}
\Xi^{(p)}\left(x_{1}, \ldots, x_{d}\right)= & \sqrt{\Sigma\left(x_{1}, \ldots, x_{d}\right)} \\
\cdot & \int_{\Omega_{d}} G\left(x_{1}, \ldots, x_{d} ; y_{1}, \ldots, y_{d}\right) \sqrt{\Sigma\left(y_{1}, \ldots, y_{d}\right)} \Xi^{(p-1)}\left(y_{1}, \ldots, y_{d}\right) d^{d} y
\end{aligned}
$$

converges to the lowest mode of Eq.(1) having an overlap with $\Xi^{(0)}$; in particular, if $\Xi^{(0)}$ has an overlap with the fundamental mode of Eq. (1) one has

$$
\Psi_{0}\left(x_{1}, \ldots, x_{d}\right)=\lim _{p \rightarrow \infty} \frac{\Xi^{(p)}\left(x_{1}, \ldots, x_{d}\right)}{\sqrt{\Sigma\left(x_{1}, \ldots, x_{d}\right)}}
$$

Proof. The proof is simple: we may cast Eq.(1) in the form

$$
\frac{1}{\sqrt{\Sigma\left(x_{1}, \ldots, x_{d}\right)}}\left(-\Delta_{d}\right) \frac{1}{\sqrt{\Sigma\left(x_{1}, \ldots, x_{d}\right)}} \Phi_{n}\left(x_{1}, \ldots, x_{d}\right)=E_{n} \Phi_{n}\left(x_{1}, \ldots, x_{d}\right)
$$

where $\Psi_{n}\left(x_{1}, \ldots, x_{d}\right)=\Phi_{n}\left(x_{1}, \ldots, x_{d}\right) / \sqrt{\Sigma\left(x_{1}, \ldots, x_{d}\right)}$. Notice that Eq.(1) and (4) are isospectral and call $\hat{O}$ the operator appearing on the left hand side of the equation

$$
\hat{O} \equiv \frac{1}{\sqrt{\Sigma\left(x_{1}, \ldots, x_{d}\right)}}\left(-\Delta_{d}\right) \frac{1}{\sqrt{\Sigma\left(x_{1}, \ldots, x_{d}\right)}}
$$

The spectrum of $\hat{O}$ is bounded from below and contains only positive eigenvalues; the ansatz $\Xi^{(0)}\left(x_{1}, \ldots, x_{d}\right)$ can be decomposed in the basis of the eigenfunctions of Eq.(4)

$$
\Xi^{(0)}\left(x_{1}, \ldots, x_{d}\right)=\sum_{n} c_{n} \Phi_{n}\left(x_{1}, \ldots, x_{d}\right)
$$

Now notice that Eq.(2) may be written as

$$
\Xi^{(p)}=\hat{O}^{-1} \Xi^{(p-1)}
$$

where

$$
\hat{O}^{-1} \equiv \sqrt{\Sigma\left(x_{1}, \ldots, x_{d}\right)}\left(\begin{array}{c}
\left.-\Delta_{d}\right)^{-1} \\
3
\end{array}\right.
$$


is the inverse operator $\left(\hat{O} \hat{O}^{-1}=\hat{O}^{-1} \hat{O}=\mathbf{1}\right)$.

The spectrum of $\hat{O}^{-1}$ is positive and bounded both from below and from above: therefore after $p$ repeated applications of Eq.(6) one obtains

$$
\Xi^{(p)}\left(x_{1}, \ldots, x_{d}\right)=\sum_{n} \frac{c_{n}}{E_{n}^{p}} \Phi_{n}\left(x_{1}, \ldots, x_{d}\right)
$$

In the limit $p \rightarrow \infty$ only the term with lowest eigenvalue of $\hat{O}$ with non-vanishing coefficient $c_{n}$ survives; this proves the theorem.

We need to discuss separately the case of a spectrum containing a zero mode (in one dimension this case corresponds to Neumann or periodic boundary conditions).

Theorem 2. (Spectrum containing a zero mode) We assume that the spectrum of Eq. (I) contains a zero mode. Consider a d dimensional region of space, $\Omega_{d}$, and a density $\Sigma\left(x_{1}, \ldots, x_{d}\right)>0$ which varies over $\Omega_{d}$ and let $\Xi^{(0)}\left(x_{1}, \ldots, x_{d}\right)$ be an arbitrary function defined on $\Omega_{d}$, which has an overlap with the lowest mode of the Helmholtz equation (I) with positive eigenvalue.

Let $G^{(0)}\left(x_{1}, \ldots, x_{d} ; y_{1}, \ldots, y_{d}\right)$ be the "regularized" Green's function of the homogeneous problem on $\Omega_{d}$, obeying the same boundary conditions of $\Psi$. We call $\Psi_{0}\left(x_{1}, \ldots, x_{d}\right)$ the eigenfunction of the zero mode, corresponding to the vanishing eigenvalue, and $\Psi_{1}\left(x_{1}, \ldots, x_{d}\right)$ the eigenfunction of the mode with smallest positive eigenvalue.

Then, for $p \rightarrow \infty$, the sequence of functions

$$
\begin{aligned}
\tilde{\Xi}^{(p)}\left(x_{1}, \ldots, x_{d}\right)= & \sqrt{\Sigma\left(x_{1}, \ldots, x_{d}\right)} \\
& \cdot \int_{\Omega_{d}} G^{(0)}\left(x_{1}, \ldots, x_{d} ; y_{1}, \ldots, y_{d}\right) \sqrt{\Sigma\left(y_{1}, \ldots, y_{d}\right)} \Xi^{(p-1)}\left(y_{1}, \ldots, y_{d}\right) d^{d} y(9) \\
\Xi^{(p)}\left(x_{1}, \ldots, x_{d}\right)= & \tilde{\Xi}^{(p)}\left(x_{1}, \ldots, x_{d}\right) \\
- & \sqrt{\Sigma\left(x_{1}, \ldots, x_{d}\right)} \frac{\int_{\Omega_{d}} \sqrt{\sum\left(y_{1}, \ldots, y_{d}\right)} \tilde{\Xi}^{(p)}\left(y_{1}, \ldots, y_{d}\right) d^{d} y}{\int_{\Omega_{d}} \Sigma\left(y_{1}, \ldots, y_{d}\right) d^{d} y}
\end{aligned}
$$

converges to the lowest mode of Eq.(I) with positive eigenvalue having an overlap with $\Xi^{(0)}$; in particular, if $\Xi^{(0)}$ has an overlap with the the mode with smallest positive eigenvalue one has

$$
\Psi_{1}\left(x_{1}, \ldots, x_{d}\right)=\lim _{p \rightarrow \infty} \frac{\Xi^{(p)}\left(x_{1}, \ldots, x_{d}\right)}{\sqrt{\Sigma\left(x_{1}, \ldots, x_{d}\right)}}
$$

Proof. We need to generalize the previous proof. First we notice that the explicit form of the eigenfunction of the zero mode is known:

$$
\Psi_{0}\left(x_{1}, \ldots, x_{d}\right)=1 / \sqrt{V_{\Omega}}
$$

where $V_{\Omega}$ is the volume of $\Omega_{d}$. This implies that

$$
\Phi_{0}\left(x_{1}, \ldots, x_{d}\right)=\sqrt{\frac{\sum\left(x_{1}, \ldots, x_{d}\right)}{V_{\Omega}}}
$$

As we have discussed in Ref. [18], it is convenient to define the modified operator

$$
\hat{O}_{\gamma} \equiv \frac{1}{\sqrt{\Sigma\left(x_{1}, \ldots, x_{d}\right)}}(-\Delta+\gamma) \frac{1}{\sqrt{\Sigma\left(x_{1}, \ldots, x_{d}\right)}}
$$


where $\gamma \rightarrow 0^{+}$; similarly we modify Eq. (1) as

$$
(-\Delta+\gamma) \Psi_{n}\left(x_{1}, \ldots, x_{d}\right)=E_{n} \Sigma\left(x_{1}, \ldots, x_{d}\right) \Psi_{n}\left(x_{1}, \ldots, x_{d}\right)
$$

The presence of a zero mode is now avoided, as long as $\gamma>0$.

The Green's function is now

$$
G_{\gamma}\left(x_{1}, \ldots, x_{d}, y_{1}, \ldots, y_{d}\right)=\frac{1}{V_{\Omega} \gamma}+\sum_{n}^{\prime} \frac{\phi_{n}\left(x_{1}, \ldots, x_{d}\right) \phi_{n}\left(y_{1}, \ldots, y_{d}\right)}{\epsilon_{n}+\gamma}
$$

where $V_{\Omega}$ is the volume of the region $\Omega$. Here $\epsilon_{n}$ and $\phi_{n}$ are the eigenvalues and eigenfunctions of the negative Laplacian on $\Omega$ obeying specific boundary conditions and $\sum_{n}^{\prime}$ is the sum over all possible modes, with the exclusion of the zero mode.

In particular, in one dimension the Green's function can be expressed in a simple form which reads

$$
G_{\gamma}(x, y)=\left\{\begin{array}{lll}
\frac{\left(e^{\sqrt{\gamma}(a+2 x)}-1\right)\left(e^{a \sqrt{\gamma}}-e^{2 \sqrt{\gamma} y}\right) \operatorname{csch}(a \sqrt{\gamma}) e^{-\sqrt{\gamma}(a+x+y)}}{4 \sqrt{\gamma}} & , \quad-a / 2 \leq x<y \leq a / 2 \\
\frac{\left(e^{a \sqrt{\gamma}}-e^{2 \sqrt{\gamma} x}\right)\left(e^{\sqrt{\gamma}(a+2 y)}-1\right) \operatorname{csch}(a \sqrt{\gamma}) e^{-\sqrt{\gamma}(a+x+y)}}{4 \sqrt{\gamma}} & , \quad-a / 2 \leq y<x \leq a / 2
\end{array}\right.
$$

It is possible to expand the Green's function around $\gamma=0$ as

$$
G_{\gamma}\left(x_{1}, \ldots, x_{d}, y_{1}, \ldots, y_{d}\right)=\frac{1}{V_{\Omega} \gamma}+\sum_{q=0}^{\infty}(-1)^{q} \gamma^{q} G^{(q)}(x, y)
$$

where

$$
G^{(q)}(x, y) \equiv \sum_{n \neq 0} \frac{\phi_{n}\left(x_{1}, \ldots, x_{d}\right) \phi_{n}\left(y_{1}, \ldots, y_{d}\right)}{\epsilon_{n}^{q+1}}
$$

Observe that $G^{(0)}(x, y)$ is the "regularized" Green's function discussed in [19, 20].

At this point, we have converted our problem to the same form of the case discussed before; the application of Theorem 1 using the shifted Green's function $G_{\gamma}\left(x_{1}, \ldots, x_{d}, y_{1}, \ldots, y_{d}\right)$ could therefore be used to obtain the power series expansion of the eigenvalue and of the eigenfunction of the fundamental mode around $\gamma=0$. However, while this calculation is useful in the evaluation of the sum rules involving eigenvalues of $\hat{O}$ (see the discussion in [18]), in the present case we are interested in calculating the first excited state of $\hat{O}$.

Let us first apply Theorem 1 to the shifted problem writing

$$
\begin{aligned}
\tilde{\Xi}^{(1)}\left(x_{1}, \ldots, x_{d}\right)= & \sqrt{\Sigma\left(x_{1}, \ldots, x_{d}\right)} \\
& \cdot \int_{\Omega_{d}} G_{\gamma}\left(x_{1}, \ldots, x_{d} ; y_{1}, \ldots, y_{d}\right) \sqrt{\Sigma\left(y_{1}, \ldots, y_{d}\right)} \Xi^{(0)}\left(y_{1}, \ldots, y_{d}\right) d^{d} y
\end{aligned}
$$

Assuming that the starting ansatz is independent of $\gamma$, one should realize that that $\tilde{\Xi}^{(1)}\left(x_{1}, \ldots, x_{d}\right)$ now contains terms which depend on $1 / \gamma$. These terms, however, are only present if $\tilde{\Xi}^{(0)}\left(x_{1}, \ldots, x_{d}\right)$ is not orthogonal to $\Phi_{0}\left(x_{1}, \ldots, x_{d}\right)$. The effect of orthogonalizing $\tilde{\Xi}^{(1)}\left(x_{1}, \ldots, x_{d}\right)$ with respect to $\Phi_{0}\left(x_{1}, \ldots, x_{d}\right)$ is then to eliminate the factors which depend on $1 / \gamma$. Moreover, if these terms are not present, one can then work with the regularized Green's function $G^{(0)}$ rather than the full Green's function: as a matter of fact the only way that the infinitesimal contributions of $G^{(q)}$, 
with $q=1,2, \ldots$, could survive for $\gamma \rightarrow 0^{+}$would be if they could interfere with divergent contributions going as $1 / \gamma^{q}$, which however are absent because of the orthogonalization.

As a result we can write Eq.(20) in terms of the regularized Green's function $G^{(0)}$ and Eqs.(9) and (10) follow.

At each iteration the lowest energy components of the ansatz are inflated, and the component corresponding to the zero mode is removed by orthogonalization. Therefore, we have

$$
\Psi_{1}\left(x_{1}, \ldots, x_{d}\right)=\lim _{p \rightarrow \infty} \frac{\Xi^{(p)}\left(x_{1}, \ldots, x_{d}\right)}{\sqrt{\sum\left(x_{1}, \ldots, x_{d}\right)}}
$$

This proves the theorem.

Remark 1. Theorems 1 and 2 are the extension of the Power Method (PM) for approximating eigenvalues and eigenvectors of a finite hermitian matrix to the case of a hermitian operator (and therefore to the infinite dimensional case). This observation was already made in refs. [21, 22] for the analogous results discussed there.

As for the case of the Power Method, the rate of convergence of the iterated function towards the eigenfunction of the fundamental mode of Eq.(1) is essentially determined by the separation between the two largest eigenvalues of $\hat{O}^{-1}$.

The results contained in Refs. [22] and [21] were limited to Dirichlet boundary conditions and were not formulated explicitly in terms of Green's functions. It is convenient to consider this alternative approach in more detail: as we have discussed in Ref. [23] it is possible to cast eq.(1) into the alternative form

$$
\left(-\Delta_{d}\right)^{1 / 2} \frac{1}{\Sigma\left(x_{1}, \ldots, x_{d}\right)}\left(-\Delta_{d}\right)^{1 / 2} \Xi_{n}\left(x_{1}, \ldots, x_{d}\right)=E_{n} \Xi_{n}\left(x_{1}, \ldots, x_{d}\right)
$$

where $\Xi_{n}\left(x_{1}, \ldots, x_{d}\right) \equiv\left(-\Delta_{d}\right)^{1 / 2} \Psi_{n}\left(x_{1}, \ldots, x_{d}\right)$. Notice that Eqs.(1), (4) and (22) are isospectral. Following the notation of Ref. [23] we define the operator

$$
\hat{W} \equiv\left(-\Delta_{d}\right)^{1 / 2} \frac{1}{\Sigma\left(x_{1}, \ldots, x_{d}\right)}\left(-\Delta_{d}\right)^{1 / 2}
$$

Clearly the inverse operator is

$$
\hat{W}^{-1} \equiv\left(-\Delta_{d}\right)^{-1 / 2} \Sigma\left(x_{1}, \ldots, x_{d}\right)\left(-\Delta_{d}\right)^{-1 / 2}
$$

Therefore, the implementation of Theorem 1 in matrix form may be done either the matrix elements of either operators $\hat{O}^{-1}$ or $\hat{W}^{-1}$. The obvious choice is to use a basis of eigenstates of the Laplacian operator (we call $\left|n_{1}, \ldots, n_{d}\right\rangle$ and $\epsilon_{n_{1}, \ldots, n_{d}}$ the eigenstates and eigenvalues of the Laplacian respectively) and therefore

$$
\begin{aligned}
\left\langle n_{1}, \ldots, n_{d}\left|\hat{O}^{-1}\right| m_{1}, \ldots, m_{d}\right\rangle & =\sum_{r_{1}, \ldots, r_{d}} \frac{\left\langle n_{1}, \ldots, n_{d}|\sqrt{\Sigma}| r_{1}, \ldots, r_{d}\right\rangle\left\langle r_{1}, \ldots, r_{d}|\sqrt{\Sigma}| m_{1}, \ldots, m_{d}\right\rangle}{\epsilon_{r_{1}, \ldots, r_{d}}} \\
\left\langle n_{1}, \ldots, n_{d}\left|\hat{W}^{-1}\right| m_{1}, \ldots, m_{d}\right\rangle & =\frac{\left\langle n_{1}, \ldots, n_{d}|\Sigma| m_{1}, \ldots, m_{d}\right\rangle}{\sqrt{\epsilon_{n_{1}, \ldots, n_{d}}} \sqrt{\epsilon_{m_{1}, \ldots, m_{d}}}}
\end{aligned}
$$

Working with a finite portion of the Hilbert space, containing the lowest $N$ states, one obtains approximate values for the eigenvalues and eigenstates of the operators in terms of the corresponding quantities of the finite matrices. This approach amounts to applying the Rayleigh-Ritz 
method to this problem. Although for $N \rightarrow \infty$ the two matrices must yield the same eigenvalues, the approximations obtained with a finite portion of the Hilbert space are necessarily poorer in the first case where the matrix element contains a sum over internal quantum numbers.

The matrix form of Theorem 1 corresponds to applying the power method directly to the $N \times N$ matrices discussed above, picking an arbitrary trial vector. This approach only requires matrix vector multiplications, and therefore it can be efficiently implemented in a computer, with a minimal use of memory.

Remark 2. These theorems apply to arbitrary regions in $d$-dimensions, although they require the knowledge of the Green's function of the homogeneous problem corresponding to that region, and therefore of the spectrum of the associated homogeneous problem. In two dimensions, however, one can always reduce the original problem to an equivalent problem over a suitable region (i.e. a region where a basis of functions is known) with the use of an appropriate conformal map.

We briefly discuss this point: consider an arbitrary simply connected region of the plane, $\Gamma$, where the Helmholtz equation

$$
-\left(\frac{\partial^{2}}{\partial u^{2}}+\frac{\partial^{2}}{\partial v^{2}}\right) \psi_{n}(u, v)=E_{n} \psi_{n}(u, v)
$$

is fulfilled. Now let $w \equiv u+i v=f(z)$ map conformally a region $\Omega$ of the plane where the Green's function of the homogeneous problem is known (rectangle, circle, ...) to $\Gamma$ : on $\Omega$ we have

$$
-\left(\frac{\partial^{2}}{\partial x^{2}}+\frac{\partial^{2}}{\partial y^{2}}\right) \Psi_{n}(x, y)=E_{n} \Sigma(x, y) \Psi_{n}(x, y)
$$

where $\Sigma(x, y)=\left|\frac{d f}{d z}\right|^{2}$. Therefore we may obtain the eigenfunction and eigenvalue of the fundamental mode of Eq. 25), applying Theorems 1 and 2 to Eq.(26).

In this particular case, the Rayleigh-Ritz approach with a finite number of basis functions is essentially equivalent to the "conformal mapping method" of Ref. [24] (see also Ref. [22]).

Remark 3. (Variational theorem) Let $\Xi^{(p)}\left(x_{1}, \ldots, x_{d}\right)$ be the function obtained using Theorem 1 after $p$ iterations: the expectation value of $\hat{O}$ (Rayleigh quotient) is

$$
\langle\hat{O}\rangle_{p}=\frac{\int d^{d} x \Xi^{(p)}\left(x_{1}, \ldots, x_{d}\right) \hat{O} \Xi^{(p)}\left(x_{1}, \ldots, x_{d}\right)}{\int d^{d} x\left[\Xi^{(p)}\left(x_{1}, \ldots, x_{d}\right)\right]^{2}}
$$

If $\Xi^{(0)}\left(x_{1}, \ldots, x_{d}\right)$ depends on one or more arbitrary parameters $\mu_{i}$, the minimization of $\langle\hat{O}\rangle_{p}$ with respect to these parameters leads to an optimal approximation of the eigenfunction of the fundamental mode of $\hat{O}$ in the class of functions considered.

We will now state another theorem which allows one to obtain approximations to the eigenfunction of the fundamental mode of Eq.(1). We restrict to the case in which the zero mode is not present. A generalization to the case in which the zero mode is present can be done along the lines of what done in Theorem 2 .

Theorem 3. We assume that the hypotheses of Theorem 1 hold. Let $\xi^{(0)}\left(x_{1}, \ldots, x_{d}\right)$ be an arbitrary function on $\Omega$, that we require to be normalized, $\int_{\Omega} d^{d} x \xi^{(0)}\left(x_{1}, \ldots, x_{d}\right)^{2}=1$; we adopt the Dirac bra-ket notation and work with the state $\left|\xi^{(0)}\right\rangle\left(\left\langle\xi^{(0)} \mid \xi^{(0)}\right\rangle=1\right)$; 
We define the state

$$
\left|\chi^{(0)}\right\rangle \equiv \frac{1}{v^{(0)}}\left(\hat{O}^{-1}-\eta^{(0)}\right)\left|\xi^{(0)}\right\rangle
$$

where

$$
\begin{aligned}
& \eta^{(0)} \equiv\left\langle\xi^{(0)}\left|\hat{O}^{-1}\right| \xi^{(0)}\right\rangle \\
& v^{(0)} \equiv\left\langle\xi^{(0)}\left|\hat{O}^{-1}\right| \chi^{(0)}\right\rangle=\sqrt{\left\langle\xi^{(0)}\left|\hat{O}^{-2}\right| \xi^{(0)}\right\rangle-\left(\eta^{(0)}\right)^{2}}
\end{aligned}
$$

Then, for $p \rightarrow \infty$, with $p$ integer, the state

$$
\left|\xi^{(p+1)}\right\rangle \equiv \frac{1}{\sqrt{2}}\left\{\sqrt{\frac{-\varepsilon^{(p)}+\eta^{(p)}+\Delta^{(p)}}{\Delta^{(p)}}}\left|\xi^{(p)}\right\rangle+\sqrt{\frac{\varepsilon^{(p)}-\eta^{(p)}+\Delta^{(p)}}{\Delta^{(p)}}}\left|\chi^{(p)}\right\rangle\right\}
$$

with

$$
\left|\chi^{(p)}\right\rangle \equiv \frac{1}{v^{(p)}}\left(\hat{O}^{-1}-\eta^{(p)}\right)\left|\xi^{(p)}\right\rangle
$$

and

$$
\begin{aligned}
\eta^{(p)} & \equiv\left\langle\xi^{(p)}\left|\hat{O}^{-1}\right| \xi^{(p)}\right\rangle \\
v^{(p)} & \equiv\left\langle\xi^{(p)}\left|\hat{O}^{-1}\right| \chi^{(p)}\right\rangle=\sqrt{\left\langle\xi^{(p)}\left|\hat{O}^{-2}\right| \xi^{(p)}\right\rangle-\left(\eta^{(p)}\right)^{2}} \\
\varepsilon^{(p)} & \equiv\left\langle\chi^{(p)}\left|\hat{O}^{-1}\right| \chi^{(p)}\right\rangle \\
\Delta^{(p)} & \equiv \sqrt{\left(\eta^{(p)}-\varepsilon^{(p)}\right)^{2}+\left(2 v^{(p)}\right)^{2}}
\end{aligned}
$$

converges to the eigenstate of Eq. (4) with lowest eigenvalue.

Proof. This theorem is an implementation of the Lanczos algorithm [25] to Hermitian operators in an Hilbert space. In a different context, implementations of the Lanczos algorithm are found, for example, in Refs. [26, 27, 28].

In particular, it is easy to adapt the demonstration in Ref. [26] to the present case: our demonstration differs in that the method is applied to the inverse operator $\hat{O}^{-1}$.

First we notice that $\left|\chi^{(p)}\right\rangle$ is orthogonal to $\left|\xi^{(p)}\right\rangle$ and normalized to 1 :

$$
\left\langle\xi^{(p)} \mid \chi^{(p)}\right\rangle=0 \quad, \quad\left\langle\chi^{(p)} \mid \chi^{(p)}\right\rangle=1
$$

These two states define a two-dimensional subspace and one can diagonalize $\hat{O}^{-1}$ in this space obtaining two new states

$$
\begin{aligned}
& \left|V_{1}\right\rangle \equiv \frac{1}{\sqrt{2}}\left\{-\sqrt{\frac{\varepsilon^{(p)}-\eta^{(p)}+\Delta^{(p)}}{\Delta^{(p)}}}\left|\xi^{(p)}\right\rangle+\sqrt{\frac{-\varepsilon^{(p)}+\eta^{(p)}+\Delta^{(p)}}{\Delta^{(p)}}}\left|\chi^{(p)}\right\rangle\right\} \\
& \left|V_{2}\right\rangle \equiv \frac{1}{\sqrt{2}}\left\{\sqrt{\frac{-\varepsilon^{(p)}+\eta^{(p)}+\Delta^{(p)}}{\Delta^{(p)}}}\left|\xi^{(p)}\right\rangle+\sqrt{\frac{\varepsilon^{(p)}-\eta^{(p)}+\Delta^{(p)}}{\Delta^{(p)}}}\left|\chi^{(p)}\right\rangle\right\}
\end{aligned}
$$


with corresponding eigenvalues

$$
\begin{aligned}
& \mathcal{E}_{1} \equiv \frac{1}{2}\left(\eta^{(p)}+\varepsilon^{(p)}-\Delta^{(p)}\right) \\
& \mathcal{E}_{2} \equiv \frac{1}{2}\left(\eta^{(p)}+\varepsilon^{(p)}+\Delta^{(p)}\right)
\end{aligned}
$$

Now notice that $\Delta^{(p)} \geq\left|\varepsilon^{(p)}-\eta^{(p)}\right|$ and one finds that

$$
\mathcal{E}_{1} \leq \eta^{(p)}, \quad \mathcal{E}_{2} \geq \eta^{(p)}
$$

Therefore, Eq. (31) corresponds to selecting the second eigenvector, where the expectation value of $\hat{O}^{-1}$ has increased 2 . If this process is carried out indefinitely, then $\left|\xi^{(p+1)}\right\rangle$ must converge to the lowest state of $\hat{O}$ which has a non-zero overlap with the initial ansatz.

Also in this case one can easily apply the Theorem in its matrix form, using the matrix elements of $\hat{O}^{-1}$ and $\hat{W}^{-1}$ that we have previously obtained.

Remark 4. The standard implementation of the Lanczos method would work directly with the operator $\hat{O}$. In our case, however, there are good reasons for using $\hat{O}^{-1}$ in generating the $\left\langle\chi^{(p)}\right\rangle$ : as a matter of fact, the direct application of $\hat{O}$ to an arbitrary ansatz in general does not produce a function with the same boundary conditions and therefore the method is not applicable in a straightforward manner. On the other hand, the application of $\hat{O}^{-1}$ to an arbitrary function $\psi$ produces a new function with the correct boundary conditions even in the case where the boundary conditions are not enforced on $\psi$.

Observe also that since the new ansatz is obtained diagonalizing $\hat{O}^{-1}$ in the subspace spanned by $\left|\xi^{(p)}\right\rangle$ and $\left|\chi^{(p)}\right\rangle$, one has to select the eigenstate with largest eigenvalue, in contrast with the case of Refs.[26, 27, 28], where the lowest eigenvalue is picked 3 .

We now discuss the calculation of the excited states of the Helmholtz equation (1) and prove two different theorems.

Theorem 4. (Spectrum without a zero mode) Let $\left\{\Xi_{0}^{(1)}\left(x_{1}, \ldots, x_{d}\right), \ldots, \Xi_{0}^{(N)}\left(x_{1}, \ldots, x_{d}\right)\right\}$ be a set of arbitrary functions defined on $\Omega_{d}$. We assume that these functions have a nonzero overlap with the lowest $N$ eigenfunctions of the Helmholtz equation (4) and that these are ordered such that

$$
\begin{aligned}
0< & \frac{\int_{\Omega_{d}} \Xi_{0}^{(1)}\left(x_{1}, \ldots, x_{d}\right) \hat{O} \Xi_{0}^{(1)}\left(x_{1}, \ldots, x_{d}\right) d^{d} x}{\int_{\Omega_{d}}\left[\Xi_{0}^{(1)}\left(x_{1}, \ldots, x_{d}\right)\right]^{2} d^{d} x}<\ldots< \\
< & \frac{\int_{\Omega_{d}} \Xi_{0}^{(N)}\left(x_{1}, \ldots, x_{d}\right) \hat{O} \Xi_{0}^{(N)}\left(x_{1}, \ldots, x_{d}\right) d^{d} x}{\int_{\Omega_{d}}\left[\Xi_{0}^{(N)}\left(x_{1}, \ldots, x_{d}\right)\right]^{2} d^{d} x}
\end{aligned}
$$

\footnotetext{
${ }^{2}$ Notice the difference with the standard approach, which works on the operator $\hat{O}$ and minimizes its expectation value at each iteration.

${ }^{3}$ Alternatively, one could modify the present implementation diagonalizing $\hat{O}$ in the subspace spanned by $\left|\xi^{(p)}\right\rangle$ and $\left\langle\chi^{(p)}\right\rangle$ (in this case one would pick the eigenstate with lowest eigenvalue).
} 
Consider now the new set of functions given by

$$
\begin{aligned}
\Xi_{p}^{(j)}\left(x_{1}, \ldots, x_{d}\right)= & \sqrt{\Sigma\left(x_{1}, \ldots, x_{d}\right)} \\
\cdot & \int_{\Omega_{d}} G\left(x_{1}, \ldots, x_{d} ; y_{1}, \ldots, y_{d}\right) \sqrt{\Sigma\left(y_{1}, \ldots, y_{d}\right)} \Xi_{p-1}^{(j)}\left(y_{1}, \ldots, y_{d}\right) d^{d} y
\end{aligned}
$$

with $j=1,2, \ldots, N$ and define

$$
\begin{aligned}
\tilde{\Xi}_{p}^{(j)}\left(x_{1}, \ldots, x_{d}\right) & =\Xi_{p}^{(j)}\left(x_{1}, \ldots, x_{d}\right) \\
& -\tilde{\Xi}_{p}^{(k)}\left(x_{1}, \ldots, x_{d}\right) \sum_{k=1}^{j-1} \frac{\int_{\Omega_{d}} \tilde{\Xi}_{p}^{(k)}\left(y_{1}, \ldots, y_{d}\right) \Xi_{p}^{(j)}\left(y_{1}, \ldots, y_{d}\right) d^{d} y}{\int_{\Omega_{d}}\left[\Xi_{p}^{(k)}\left(y_{1}, \ldots, y_{d}\right)\right]^{2} d^{d} y} .
\end{aligned}
$$

For $p \rightarrow \infty$ the set $\left\{\tilde{\Xi}_{p}^{(1)}\left(x_{1}, \ldots, x_{d}\right), \ldots, \tilde{\Xi}_{p}^{(N)}\left(x_{1}, \ldots, x_{d}\right)\right\}$ converges to the first lowest $N$ eigenfunctions of Eq.(4).

Proof. The proof is analogous to the proof of Theorem 2 of Ref. [21]: at each iteration the functions generated with Eq. (42) have their lower energy components inflated, since they have been obtained from the previous set applying the inverse operator. The first function of the set converges to the lowest eigenfunction, because of Theorem 1, the remaining functions are orthogonalized at each step in Eq.(43) and therefore as the number of iterations grows they must converge to the exact eigenfunctions of the excited modes.

Remark 5. The generalization of this theorem to the case in which a zero mode is present can be done straightforwardly, along the lines discussed in Theorem 2 . Notice however that this theorem is useful only for the low lying states of an operator, since the approximate eigenfunction of the targeted state is obtained via iteration and orthogonalization with respect to the lower states. An alternative approach would be modifying Theorem 3 , applying it to the operator $\hat{Q} \equiv\left(\hat{O}^{-1}-\frac{1}{\Lambda}\right)^{2}$. This is essentially the "folded spectrum method" (FSM) of Refs. [29, 30]. However, this implementation of the FSM would not be of practical use since the spectrum of the operator $\hat{Q}$, in the part corresponding to the highly excited states of $\hat{O}$, will consist of finely spaced eigenvalues, with a separation which tends to zero for arbitrarily high states. As a result the convergence of the method is expected to be extremely slow for these states.

\section{Applications}

We will discuss some applications of the theorems that we have proved in this paper. The first two applications, in one and two dimensions, are simple, but serve to illustrate the methods discussed in this paper to high orders, obtaining highly precise results; the third application, which concerns one dimensional heterogeneous systems with an highly oscillatory density and different boundary conditions, will allow us to illustrate the effectiveness of our approach.

\subsection{A string with parabolic density}

Following Ref. [21] we consider a string with the density

$$
\Sigma(x)=(1+\alpha x)^{2}, \quad|x| \leq a / 2
$$


with $\alpha \leq 2 / a$. The choice of this particular density is dictated uniquely by the need of illustrating the implementation of our theorems, limiting the technical difficulties. In our calculations we will assume $a=1$.

We first consider the case of Dirichlet boundary conditions and we choose the ansatz

$$
\xi^{(0)}(x)=\mathcal{N} \sqrt{\Sigma(x)} \psi_{1}^{(D D)}(x)
$$

where

$$
\mathcal{N}=\frac{2 \sqrt{3}}{\sqrt{\left(1-\frac{6}{\pi^{2}}\right) \alpha^{2}+12}}
$$

is a normalization constant and $\psi_{n}^{(D D)}(x)$ are the Dirichlet eigenfunctions of the negative Laplacian on $|x| \leq a / 2$

$$
\psi_{n}^{(D D)}(x)=\sqrt{\frac{2}{a}} \sin \frac{n \pi(x+a / 2)}{a}
$$

The corresponding eigenvalues are

$$
\epsilon_{n}^{(D D)}=\frac{n^{2} \pi^{2}}{a^{2}}
$$

The Green's function in this case reads [19]

$$
G^{(D D)}(x, y)=\frac{(a-2 \max (x, y))(a+2 \min (x, y))}{4 a}
$$

After one iteration of Theorem 1 we obtain

$$
\begin{aligned}
\xi^{(1)}(x) & =\frac{\sqrt{2}}{\pi^{4}} \mathcal{N} \sqrt{\Sigma(x)}\left(-6 \alpha^{2} \cos (\pi x)+2 \pi \alpha(-2(\alpha x+1) \sin (\pi x)+\alpha+4 x)\right. \\
& \left.+(\pi \alpha x+\pi)^{2} \cos (\pi x)\right)
\end{aligned}
$$

It is straightforward to carry out the iterations to larger orders, although the explicit form of the functions $\xi^{(p)}(x)$ becomes lengthy and therefore we do not report them here. The energy of the fundamental mode may be approximated using the variational estimate of Eq. (27).

The expressions obtained using the variational formula take the form of a Padé approximant in terms of the parameter $\alpha$ of the density; for instance

$$
\begin{aligned}
\langle\hat{O}\rangle_{1} & =\frac{\mathcal{A}_{1}}{\mathcal{B}_{1}} \\
\mathcal{A}_{1} & \equiv\left(-57120 \pi^{4}+5040 \pi^{6}+84 \pi^{8}\right) \alpha^{4} \\
& +\left(-860160 \pi^{4}+60480 \pi^{6}+3360 \pi^{8}\right) \alpha^{2}+6720 \pi^{8} \\
\mathcal{B}_{1} & \equiv\left(5528880-652680 \pi^{2}+9226 \pi^{4}+15 \pi^{6}\right) \alpha^{6} \\
& +\left(82575360-11702880 \pi^{2}+326032 \pi^{4}+1260 \pi^{6}\right) \alpha^{4} \\
& +\left(-5160960 \pi^{2}+451360 \pi^{4}+8400 \pi^{6}\right) \alpha^{2}+6720 \pi^{6}
\end{aligned}
$$


We do not report the similar expressions for higher orders, which we have explicitly calculated, because of their length.

In Table 1 we display the approximate values of the fundamental eigenvalue, calculated to different orders with the aid of Theorem 1 , using the ansatz (45). The remaining rows contain the values obtained applying repeated Shanks transformations to these values. In particular, notice that the values corresponding to $\alpha=1$ agree with the value obtained in Ref. [21], applying Theorem 1 of that paper (which is a special case of our Theorem 1) to order 20.

We have also applied Theorem 3 to the same ansatz and after one iteration we have obtained an explicit approximation to the fundamental eigenvalue. In this case we have $E_{0}^{\alpha=1 / 2} \approx$ $9.687015834, E_{0}^{\alpha=1} \approx 9.191446083$ and $E_{0}^{\alpha=2} \approx 7.733951650$. Notice that these values are slightly more precise than the values obtained after two iterations of Theorem 1 (however a fair comparison between the two must take into account the different computational cost of each iteration in the two cases).

As we have already mentioned, the choice of a suitable ansatz may reduce the number of iterations needed to reach a given accuracy: in some cases, however, it may be more convenient to pick a less precise ansatz if its analytical form allows one to carry out the iterations explicitly to large orders. For instance, we may choose the simple ansatz

$$
\xi^{(0)}(x)=\frac{\sqrt{105}}{8}(2 x+1)\left(1-4 x^{2}\right)
$$

and iterate it to obtain approximations to the lowest eigenvalue of the string (44) for $\alpha=2$. In this case, it is straightforward to perform a very large number of iterations, because of the polynomial form of the functions involved, and we have easily calculated 105 iterations, obtaining an estimate for the energy of the fundamental mode which is accurate to about 130 digits. Using the same ansatz we have also applied Theorem 3 , with 70 iterations.

In Fig 1 we have plotted $\left|E_{0}^{(p)}-E_{0}^{(e x a c t)}\right|$ obtained after $p$ iterations of Theorem 1 (solid red curve) and of Theorem 3 (dotted orange line). The initial ansatz of Eq. (48) is used. The remaining curves are obtained from repeated Shanks transformations of the first set of results (from top to bottom the number of Shanks transformations increases). The exact value $E_{0}^{(\text {exact })}$ has been approximated with the most precise value obtained after 100 iterations of Theorem[1]

$$
\begin{aligned}
E_{0}^{(\text {exact }) \approx} & 7.733336533465966863902638033367838303091611969871617630 \\
& 20525195744620997306947223596884733603198306461387550007 \\
& 5565385500030558828 \ldots
\end{aligned}
$$

There are some important observations to make: it is clear that the rate of convergence of Theorem 3 is larger than the one of Theorem 1 . However, the computational cost of each iteration in the two approaches is different, since Theorem 3 requires the evaluation of $\hat{O}^{-1} \xi$ and $\hat{O}^{-2} \xi$ (and the subsequent evaluation of overlap integrals involving these functions). A second observation concerns the choice of the ansatz in the two approaches: in the application of Theorem 1 the ansatz is arbitrary and it does not even has to obey the boundary conditions (as a matter of fact $O^{-1} \xi$ obeys the boundary conditions regardless that $\xi$ does); on the other hand the ansatz needs to obey the boundary conditions when one applies Theorem 3 , since the new function is built as a linear combination of $\xi$ and $O^{-1} \xi$. A final observation regards the use of the Shanks transformation to extract highly precise results from a sequence of iterations: as one sees from the plot, the application of the Shanks transformation leads to a consistent gain in convergence, which can 
Table 1: Energy of the fundamental mode of the string with density 44 for different values of $\alpha$ using Theorem 1 The terms $s_{j}^{(n)}$ are the values obtained after $n$ repeated Shanks transformations.

\begin{tabular}{|c|c|c|c|}
\hline & $\alpha=1 / 2$ & $\alpha=1$ & $\alpha=2$ \\
\hline$\langle\hat{O}\rangle_{1}$ & 9.69310365089956 & 9.21037410544234 & 7.76924315857119 \\
$\langle\hat{O}\rangle_{2}$ & 9.68737359122776 & 9.19238760347104 & 7.73502742410347 \\
$\langle\hat{O}\rangle_{3}$ & 9.68702664891476 & 9.19138111461443 & 7.73341903683425 \\
$\langle\hat{O}\rangle_{4}$ & 9.68700556297997 & 9.19132401578685 & 7.73334058319427 \\
$\langle\hat{O}\rangle_{5}$ & 9.68700428051026 & 9.19132076814843 & 7.73333673246089 \\
$\langle\hat{O}\rangle_{6}$ & 9.68700420249783 & 9.19132058333956 & 7.73333654324603 \\
$\langle\hat{O}\rangle_{7}$ & 9.68700419775222 & 9.19132057282190 & 7.73333653394665 \\
$\langle\hat{O}\rangle_{8}$ & 9.68700419746353 & 9.19132057222331 & 7.73333653348959 \\
$\langle\hat{O}\rangle_{9}$ & 9.68700419744597 & 9.19132057218925 & 7.73333653346713 \\
$\langle\hat{O}\rangle_{10}$ & 9.68700419744490 & 9.19132057218731 & 7.73333653346602 \\
\hline$s_{1}^{(1)}$ & 9.68700428845695 & 9.19132145507167 & 7.73333970165889 \\
$s_{2}^{(1)}$ & 9.6870041985241 & 9.19132058171233 & 7.73333656016117 \\
$s_{3}^{(1)}$ & 9.6870041974577 & 9.191320572291 & 7.7333365336999 \\
$s_{4}^{(1)}$ & 9.68700419744499 & 9.19132057218833 & 7.73333653346805 \\
$s_{5}^{(1)}$ & 9.68700419744484 & 9.1913205721872 & 7.73333653346599 \\
$s_{6}^{(1)}$ & 9.68700419744483 & 9.19132057218719 & 7.73333653346597 \\
$s_{7}^{(1)}$ & 9.68700419744483 & 9.19132057218719 & 7.73333653346597 \\
$s_{8}^{(1)}$ & 9.68700419744483 & 9.19132057218719 & 7.73333653346597 \\
\hline$s_{1}^{(2)}$ & 9.68700419744490 & 9.19132057218826 & 7.73333653347512 \\
$s_{2}^{(2)}$ & 9.68700419744483 & 9.19132057218720 & 7.73333653346600 \\
$s_{3}^{(2)}$ & 9.68700419744483 & 9.19132057218719 & 7.73333653346597 \\
$s_{4}^{(2)}$ & 9.68700419744483 & 9.19132057218719 & 7.73333653346597 \\
$s_{5}^{(2)}$ & 9.68700419744483 & 9.19132057218719 & 7.73333653346597 \\
$s_{6}^{(2)}$ & 9.68700419744483 & 9.19132057218719 & 7.73333653346597 \\
\hline$s_{1}^{(3)}$ & 9.68700419744483 & 9.19132057218719 & 7.73333653346597 \\
$s_{2}^{(3)}$ & 9.68700419744483 & 9.19132057218719 & 7.73333653346597 \\
$s_{3}^{(3)}$ & 9.68700419744483 & 9.19132057218719 & 7.73333653346597 \\
$s_{4}^{(3)}$ & 9.68700419744483 & 9.19132057218719 & 7.73333653346597 \\
\hline & & & \\
\hline
\end{tabular}




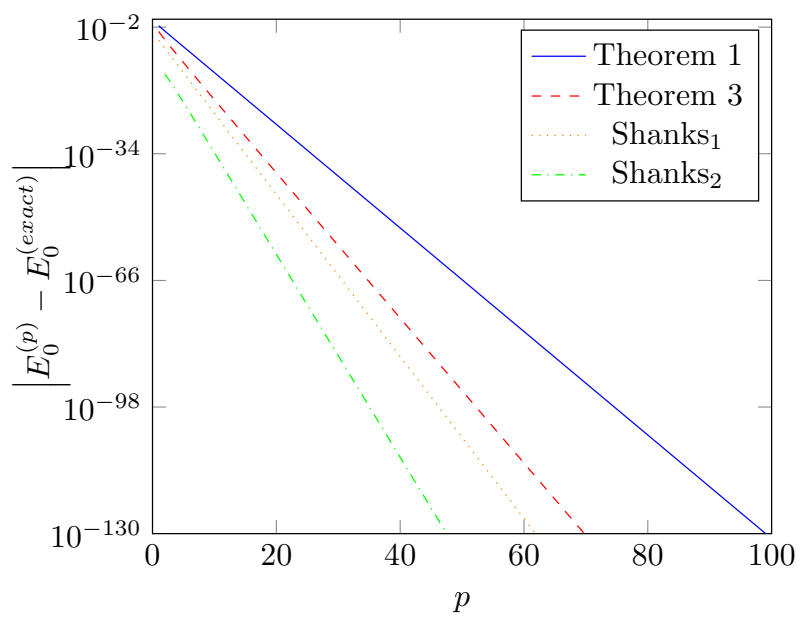

Figure 1: $\left|E_{0}^{(p)}-E_{0}^{(\text {exact })}\right|$ for the string with density $\Sigma(x)=(1+2 x)^{2}$ and Dirichlet boundary conditions obtained after $p$ iterations of Theorem 1 (solid red curve) and of Theorem 3 (dotted orange line). The initial ansatz 48 is used. The remaining curves are obtained from one and two Shanks transformations of the first set of results.

be increased repeating the transformation a number of times 4 . Since the results obtained from Theorem 1 and Theorem 3 are analytical and all integrals are calculated exactly, one does not have to worry about the insurgence of round-off errors which eventually would dominate in a purely numerical calculation. Of course, the ability of performing a large number of iterations in the application of our theorem depends on the form of the density, on the ansatz picked and ultimately on the dimensionality of the problem.

We will now consider the same string, but subject to Neumann boundary conditions. We set $\alpha=2$ and use the initial ansatz

$$
\xi^{(0)}(x)=(2 x+1)
$$

In Fig 2 we have plotted $\left|E_{0}^{(p)}-E_{0}^{(\text {exact })}\right|$ obtained after $p$ iterations of Theorem 2 (solid red curve). The remaining curves are obtained from repeated Shanks transformations of the first set of results (from top to bottom the number of Shanks transformations increases). The exact value $E_{0}^{(e x a c t)}$ has been approximated with the most precise value obtained after 105 iterations of Theorem 2 ,

$$
\begin{aligned}
E_{0}^{(\text {exact }) \approx} & 12.18713946809512900475057235600396744072004951834 \\
& 58591999323057450689688696123459800390650121512407 \\
& 90737876149983 \ldots
\end{aligned}
$$

where all the digits are correct.

\footnotetext{
${ }^{4}$ We have applied this technique in Ref. [19] to approximate the fundamental eigenvalue of one dimensional systems using the exact sum rules associated to the problem.
} 


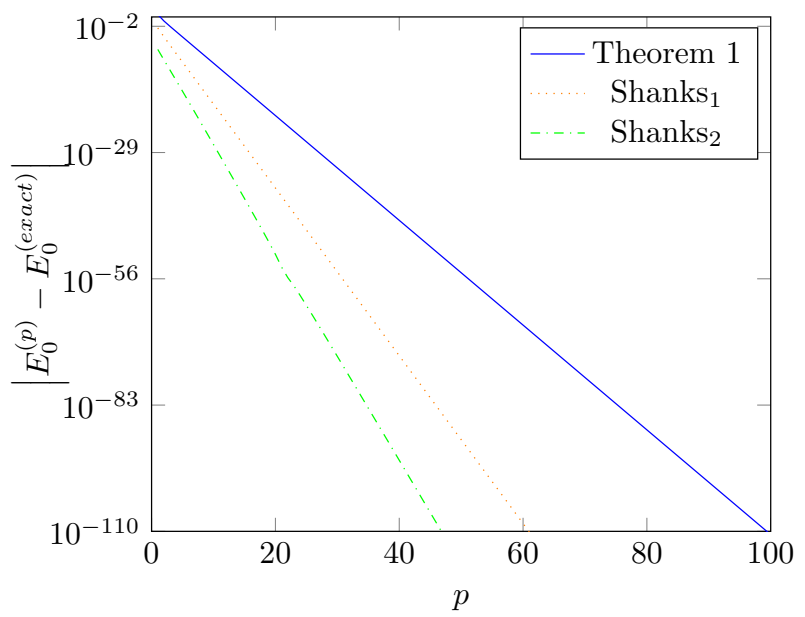

Figure 2: $\left|E_{0}^{(p)}-E_{0}^{(\text {exact })}\right|$ for the string with density $\Sigma(x)=(1+2 x)^{2}$ and Neumann boundary conditions obtained after $p$ iterations of Theorem 2 (solid red curve). The initial ansatz 49] is used. The remaining curves are obtained from one and two Shanks transformations of the first set of results.

We have also done a similar calculation for the case of periodic boundary conditions: we set $\alpha=2$ and use the same initial ansatz of Eq. (49). In Fig 3 we have plotted $\left|E_{0}^{(p)}-E_{0}^{(\text {exact })}\right|$ obtained after $p$ iterations of Theorem 2 (solid red curve). The remaining curves are obtained from repeated Shanks transformations of the first set of results (from top to bottom the number of Shanks transformations increases). The exact value $E_{0}^{(\text {exact })}$ has been approximated with the most precise value obtained after 105 iterations of Theorem 2 .

$$
E_{0}^{(\text {exact })} \approx 26.59255582932000052713762021489743577931546820189393 \ldots
$$

where all the digits are correct.

\subsection{A rectangular drum with density which varies along one direction}

We now apply Theorem 1 to the a two dimensional problem of a rectangular region $(x, y) \in$ $(-a / 2, a / 2) \times(-b / 2, b / 2)$ with Dirichlet boundary conditions at the border and with density of Eq.(44).

The Green's function for this case is

$$
G^{(D)}\left(x, y ; x^{\prime}, y^{\prime}\right)=\sum_{n_{x}=1}^{\infty} \sum_{n_{y}=1}^{\infty} \frac{\psi_{n_{x}}^{(D)}(x) \phi_{n_{y}}^{(D)}(y) \psi_{n_{x}}^{(D)}\left(x^{\prime}\right) \phi_{n_{y}}^{(D)}\left(y^{\prime}\right)}{\epsilon_{n_{x}}^{(D)}+\eta_{n_{y}}^{(D)}} .
$$

Here $\psi_{n_{x}}(x)$ and $\phi_{n_{y}}(y)$ are the Dirichlet eigenfunction of the 1D negative Laplacian on $x \in$ $(-a / 2, a / 2)$ and $y \in(-b / 2, b / 2)$ respectively:

$$
\psi_{n_{x}}(x) \equiv \sqrt{\frac{2}{a}} \sin \left(\frac{n_{x} \pi}{a}(x+a / 2)\right), n_{x}=1,2, \ldots
$$




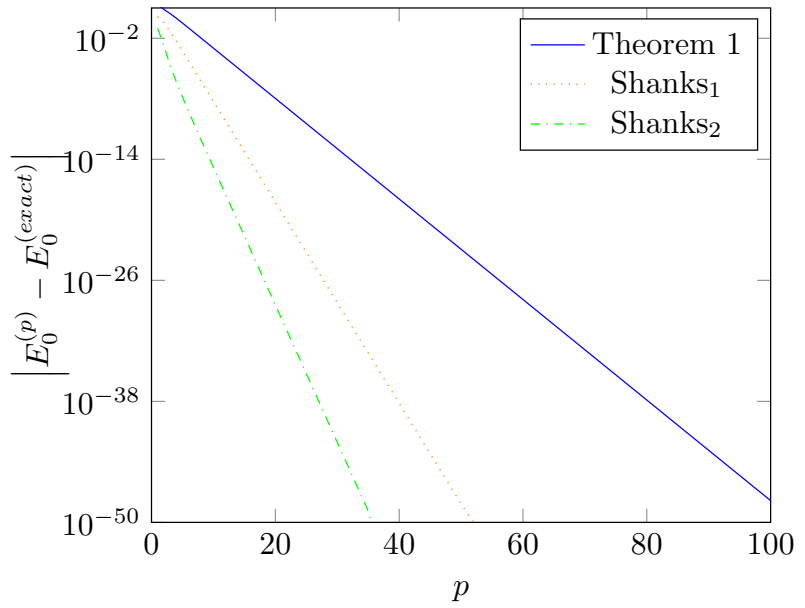

Figure 3: $\left|E_{0}^{(p)}-E_{0}^{(\text {exact })}\right|$ for the string with density $\Sigma(x)=(1+2 x)^{2}$ and periodic boundary conditions obtained after $p$ iterations of Theorem 2 (solid red curve). The initial ansatz 49 is used. The remainings curve are obtained from one and two Shanks transformations of the first set of results.

$$
\phi_{n_{y}}(y) \equiv \sqrt{\frac{2}{b}} \sin \left(\frac{n_{y} \pi}{b}(y+b / 2)\right), n_{y}=1,2, \ldots
$$

$\epsilon_{n_{x}}^{(D)}$ and $\eta_{n_{y}}^{(D)}$ are the Dirichlet eigenvalues of the 1D negative Laplacian in the two directions:

$$
\epsilon_{n_{x}}^{(D)}=\frac{n_{x}^{2} \pi^{2}}{a^{2}}, \quad \eta_{n_{y}}^{(D)}=\frac{n_{y}^{2} \pi^{2}}{b^{2}}
$$

Eq.(52) may be cast in a more convenient form as (see Refs. [31, 20])

$$
G^{(D)}\left(x, y ; x^{\prime}, y^{\prime}\right)=\sum_{n_{x}=1}^{\infty} g_{n_{x}}^{(D)}\left(y, y^{\prime}\right) \psi_{n_{x}}^{(D)}(x) \psi_{n_{x}}^{(D)}\left(x^{\prime}\right),
$$

where

$$
\begin{aligned}
g_{n_{x}}^{(D)}\left(y, y^{\prime}\right) & \equiv \sum_{n_{y}=1}^{\infty} \frac{\phi_{n_{y}}^{(D)}(y) \phi_{n_{y}}^{(D)}\left(y^{\prime}\right)}{\epsilon_{n_{x}}^{(D)}+\eta_{n_{y}}^{(D)}} \\
& =\frac{\sinh \left(\sqrt{\epsilon_{n_{x}}^{(D)}}\left(y_{<}+b / 2\right)\right) \sinh \left(\sqrt{\epsilon_{n_{x}}^{(D)}}\left(b / 2-y_{>}\right)\right)}{\sqrt{\epsilon_{n_{x}}^{(D)}} \sinh \sqrt{\epsilon_{n_{x}}^{(D)}} b} .
\end{aligned}
$$

We have introduced the notation $y_{<} \equiv \min \left(y, y^{\prime}\right)$ and $y_{>} \equiv \max \left(y, y^{\prime}\right)$. 


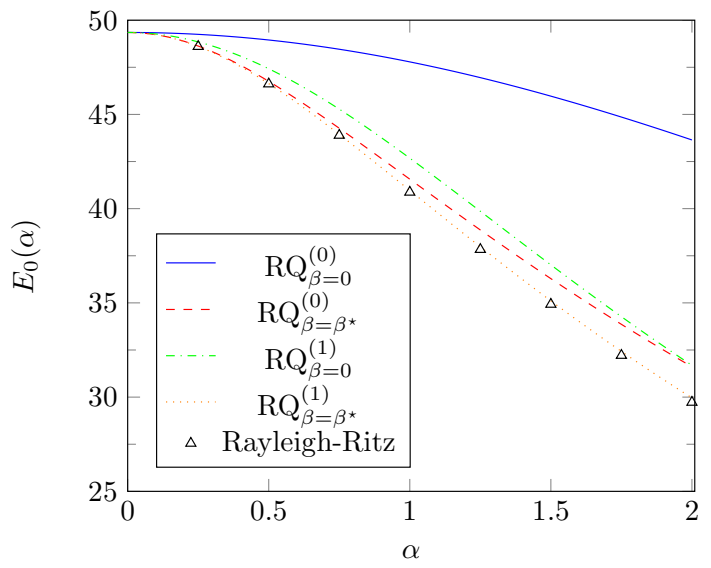

Figure 4: Approximate energy of the fundamental mode of a rectangular membrane with sides $a=1$ and $b=1 / 2$, with density (44). The solid and dashed lines are the values obtained using the bounds of eqs. 59] and 63. respectively, setting $\beta=0$. The dotted and dot-dashed lines are the values obtained using the bounds of eqs. (59) and (63) respectively, setting $\beta=\beta^{\star}$. The dots are the precise results obtained using a Rayleigh-Ritz approach with 800 basis functions.

We pick the initial ansatz

$$
\Xi^{(0)}(x, y)=(1+\beta x) \sqrt{\Sigma(x, y)} \psi_{1}^{(D)}(x) \phi_{1}^{(D)}(y)
$$

Using this ansatz we obtain the upper bound for the energy of the fundamental mode

$$
\begin{aligned}
E_{0} & \leq E_{0}^{(v a r)}(\beta)=\frac{\int \Xi^{(0)} \hat{O} \Xi^{(0)} d x d y}{\int \Xi^{(0)} \Xi^{(0)} d x d y} \\
& =\frac{20 \pi^{4}\left(6 a^{2} \beta^{2}\left(b^{2}-a^{2}\right)+\pi^{2}\left(a^{2}+b^{2}\right)\left(a^{2} \beta^{2}+12\right)\right)}{a^{2} b^{2}\left(3\left(120-20 \pi^{2}+\pi^{4}\right) a^{4} \alpha^{2} \beta^{2}+20 \pi^{2}\left(\pi^{2}-6\right) a^{2}\left(\alpha^{2}+4 \alpha \beta+\beta^{2}\right)+240 \pi^{4}\right)}
\end{aligned}
$$

$E_{0}^{(v a r)}(\beta)$ has a minimum at $\beta=\beta^{\star}$ with

$$
\beta^{\star}=\frac{45 a^{2} \alpha^{2}\left(5 a^{2}+7 b^{2}\right)+\pi^{4} a^{2} \alpha^{2}\left(a^{2}+b^{2}\right)-15 \pi^{2}\left(2 a^{4} \alpha^{2}+3 b^{2}\left(a^{2} \alpha^{2}+4\right)\right)+\sqrt{\Gamma}}{5\left(\pi^{2}-6\right) a^{2} \alpha\left(\left(\pi^{2}-6\right) a^{2}+\left(6+\pi^{2}\right) b^{2}\right)}(
$$

where

$$
\begin{aligned}
\Gamma & \equiv 300 \pi^{2}\left(\pi^{2}-6\right)^{2} a^{2} \alpha^{2}\left(a^{2}+b^{2}\right)\left(\left(\pi^{2}-6\right) a^{2}+\left(6+\pi^{2}\right) b^{2}\right) \\
& +\left(\left(\pi^{2}-15\right)^{2} a^{4} \alpha^{2}+\left(315-45 \pi^{2}+\pi^{4}\right) a^{2} \alpha^{2} b^{2}-180 \pi^{2} b^{2}\right)^{2}
\end{aligned}
$$

Therefore $E_{0}^{(v a r)}\left(\beta^{\star}\right)$ provides the optimal approximation to the fundamental eigenvalue using the trial function $\Xi^{(0)}$. 
Using Theorem 1 after one iteration we obtain the function

$$
\begin{aligned}
\Xi^{(1)}(x, y) & =(\alpha x+1) \cos \left(\frac{\pi y}{b}\right)\left\{\frac{(a b)^{3 / 2}\left(\left(\pi^{2}-6\right) a^{2} \alpha(\alpha+2 \beta)+12 \pi^{2}\right) \cos \left(\frac{\pi x}{a}\right)}{6 \pi^{4}\left(a^{2}+b^{2}\right)}\right. \\
& +(-1)^{n} a^{5 / 2} b^{3 / 2} \sum_{n=1}^{\infty}\left[\frac{a \alpha(2 n+1)(\alpha+2 \beta)}{\pi^{4} n^{2}(n+1)^{2}\left(a^{2}+(2 b n+b)^{2}\right)} \cos \left(\frac{\pi(2 n+1) x}{a}\right)\right. \\
& \left.\left.-\frac{8 n\left(\left(\pi-4 \pi n^{2}\right)^{2}\left(\alpha\left(3 a^{2} \alpha \beta+8\right)+4 \beta\right)-48 a^{2} \alpha^{2} \beta\left(4 n^{2}+1\right)\right)}{\pi^{6}\left(1-4 n^{2}\right)^{4}\left(a^{2}+4 b^{2} n^{2}\right)} \sin \left(\frac{2 \pi n x}{a}\right)\right]\right\}_{(62)}
\end{aligned}
$$

With this function we may obtain a stricter bound for the energy of the fundamental mode of the membrane:

$$
E_{0} \leq \frac{\int \Xi^{(1)} \hat{O} \Xi^{(1)} d x d y}{\int \Xi^{(1)} \Xi^{(1)} d x d y}
$$

where we do not report the explicit expressions for $\int \Xi^{(1)} \hat{O} \Xi^{(1)} d x d y$ and $\int \Xi^{(1)} \Xi^{(1)} d x d y$ because of their lengthy form.

In Fig 1 we plot the approximate energy of the fundamental mode of a rectangular membrane with sides $a=1$ and $b=1 / 2$, with density (44). The solid and dashed lines are the values obtained using the bounds of eqs. (59) and (63) respectively, setting $\beta=0$. The dotted and dotdashed lines are the values obtained using the bounds of eqs. (59) and (63) respectively, setting $\beta=\beta^{\star}$. The dots are the precise results obtained using a Rayleigh-Ritz approach with 800 basis functions. This example clearly illustrates the advantages of using a variational approach.

\subsection{A string with rapidly oscillating density}

The iterative methods that we have described in this paper can be applied to the study of inhomogeneous systems with a density which varies on a much smaller scale than the scale of the system itself. In particular we are interested in the behavior of the system when the period of the density tends to zero.

For simplicity we restrict the present discussion to one dimension and we study the Helmholtz equation

$$
-\frac{d^{2} \psi}{d x^{2}}=E_{n} \Sigma_{\epsilon}(x) \psi_{n}(x)
$$

for an inhomogeneous system with density

$$
\Sigma_{\epsilon}(x) \equiv \Sigma(x / \epsilon)
$$

where $\Sigma(x)$ is a periodic function. The eigenfunctions $\psi_{n}(x)$ obey specific boundary conditions (Dirichlet, Neumann, periodic or mixed Dirichlet-Neumann).

The physical properties of the composite materials which can be modelled by these equations will depend both on the microscale (i.e. the typical scale where the density changes) and on the macroscale of the system (i.e. the typical size of the system itself). However, a numerical study of these systems is challenging, because the size associated to the discretization of the problem 
must clearly be much smaller than the size of the microscale, thus leading to a huge number of grid points and therefore to an excessive use of computer memory. The "homogenization" method has been developed over the past forty years to obtain an effective description of these systems (see for example Refs. [15, 32, 33]), which have important applications in several areas of physics and applied mathematics.

Here we want to show that the approach described in this paper allows one to obtain systematic approximations to the lowest part of the spectrum of systems described by Eq. 64); for simplicity we consider a string of unit length with a rapidly oscillating density

$$
\Sigma_{\epsilon}(x)=2+\sin \left[\frac{2 \pi}{\epsilon}(x+\eta / 2)\right]
$$

where $\epsilon \rightarrow 0^{+}$is the parameter determining the typical size of the oscillations of the density, and $\eta$ is an arbitrary phase.

This problem has been originally studied by Castro and Zuazua in Ref. [16] for the case $\eta=1$, obtaining explicit expressions for the lowest eigenvalues of this string up to order $\epsilon^{3}$ using the WKB method: in Ref. [17], we have reproduced these results and we have obtained the fundamental eigenvalue of the string to order $\epsilon^{5}$, using the method described in Ref. [21].

\subsubsection{Dirichlet boundary conditions}

We use the same ansatz of Ref. [17]

$$
\xi^{(0)}(x)=\sqrt{\Sigma(x)} \psi_{1}^{(D D)}(x)
$$

where

$$
\psi_{n}^{(D D)}(x) \equiv \sqrt{2} \sin n \pi(x+1 / 2)
$$

are the Dirichlet eigenfunctions of the negative 1D Laplacian.

In analogy with the calculation in Ref. [17] we apply Theorem 1] and obtain an expression for the lowest eigenvalue of the string which is exact to order $\epsilon^{5}$, after two iterations:

$$
\begin{aligned}
E_{0} & \approx \frac{\pi^{2}}{2}-\frac{1}{64} \pi^{2} \epsilon^{2}+\frac{1}{4} \pi \epsilon^{3} \sin \left(\frac{\pi}{\epsilon}\right) \sin \left(\frac{\pi \eta}{\epsilon}\right)-\frac{15 \pi^{2} \epsilon^{4}}{1024} \\
& +\frac{1}{512} \pi \epsilon^{5}\left(116 \sin \left(\frac{\pi}{\epsilon}\right) \sin \left(\frac{\pi \eta}{\epsilon}\right)+5 \sin \left(\frac{2 \pi}{\epsilon}\right) \cos \left(\frac{2 \pi \eta}{\epsilon}\right)\right)+\ldots
\end{aligned}
$$

For $\eta=1$ this result reduces to the one obtained in Ref. [17]. The behavior of $E_{0}$ for three different values of $\eta$ is plotted in Fig 5

It is important to observe that the Rayleigh quotients obtained using Theorem 1 form a monotonically decreasing sequence, which converges to the exact eigenvalue of the fundamental mode as the number of the iterations goes to infinity. For this reason, if we study the Rayleigh quotient around $\epsilon=0$, we clearly have a hierarchy of contributions which correspond to the different powers of $\epsilon$ possibly multiplied by functions which are not analytical at $\epsilon=0$ but bounded (see for example eq. (69)). At a given iteration, it may happen, depending on the initial ansatz used, that the first few coefficients of the expansion have converged to the exact value: in this case, the contribution of the first term which has not yet converged must decrease at the next iteration. Since this must happen in a infinitesimal interval containing $\epsilon=0$, it means that only even terms 


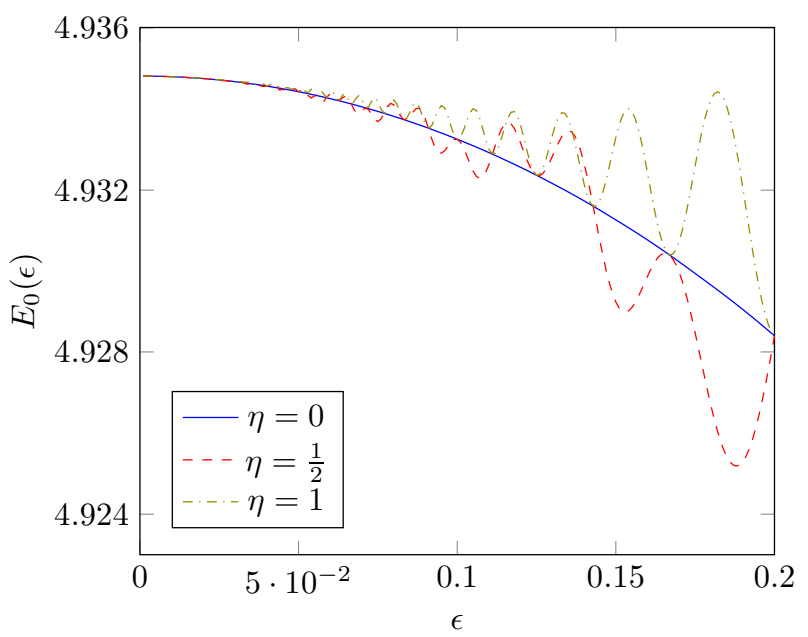

Figure 5: Eq.69 for three different values of $\eta$.

in $\epsilon$, may decrease in the whole interval: in other words, the convergence of a term which is even in $\epsilon$ forces the convergence of the subsequent term in the expansion, which is odd in $\epsilon$. This is observed in Eq. 69) which is exact to order $\epsilon^{5}$.

Alternatively, one can obtain a bound on the error on the lowest eigenvalue calculating the mean square deviation using the function obtained after $k$ iterations, $\xi^{(k)}(x)$ :

$$
\Delta^{(k)} \equiv \sqrt{\frac{\int \xi^{(k)}(x) \hat{O}^{2} \xi^{(k)}(x) d x}{\int\left[\xi^{(k)}(x)\right]^{2} d x}-\left[\frac{\int \xi^{(k)}(x) \hat{O} \xi^{(k)}(x) d x}{\int\left[\xi^{(k)}(x)\right]^{2} d x}\right]^{2}}
$$

The mean square deviations corresponding to the first two iterations are:

$$
\begin{aligned}
\Delta^{(1)} & =\frac{1}{64} \sqrt{7} \pi^{2} \epsilon^{2}+O\left[\epsilon^{3}\right] \\
\Delta^{(2)} & =\frac{\pi \epsilon^{3}}{96 \sqrt{210}}\left[\left(30240-31 \pi^{6}\right) \cos \left(\frac{2 \pi \eta}{\epsilon}\right)+\left(16 \pi^{6}-15120\right) \cos \left(\frac{2 \pi}{\epsilon}-\frac{2 \pi \eta}{\epsilon}\right)\right. \\
& \left.+\left(16 \pi^{6}-15120\right) \cos \left(\frac{2 \pi \eta}{\epsilon}+\frac{2 \pi}{\epsilon}\right)+\left(30240-31 \pi^{6}\right) \cos \left(\frac{2 \pi}{\epsilon}\right)+32 \pi^{6}-30240\right]^{1 / 2} \\
& +O\left[\epsilon^{4}\right]
\end{aligned}
$$

These functions are plotted in Fig 6

We now generalize the ansatz of eq.67) to arbitrary states:

$$
\xi_{n}^{(0)}(x)=\sqrt{\Sigma(x)} \psi_{1}^{(D D)}(x)
$$

\footnotetext{
${ }^{5}$ Clearly, Theorem 1 implies that $\lim _{k \rightarrow \infty} \Delta^{(k)}=0$.
} 


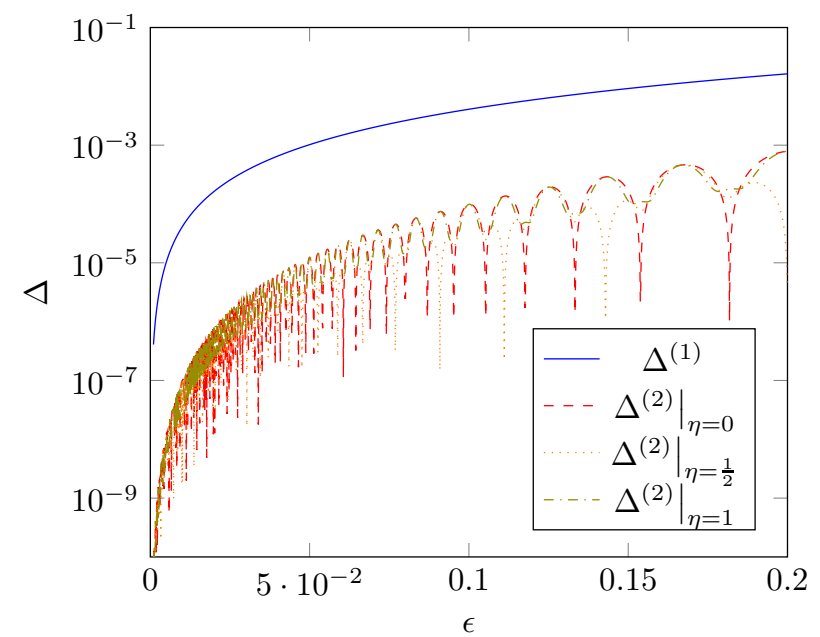

Figure 6: Mean square deviations obtained with after one and two iterations for the string with density [66 using the initial ansatz (67), with Dirichlet bc.

Although Theorem 1 only holds for the lowest state, we may check if the functions obtained after one iterations are good approximations to the excited eigenmodes for $\epsilon \rightarrow 0$; applying Eq.(2) we have

$$
\begin{aligned}
\xi_{n}^{(1)}(x) & =\sqrt{\Sigma_{\epsilon}(x)} \int_{-1 / 2}^{1 / 2} G^{(D D)}(x ; y) \sqrt{\Sigma_{\epsilon}(y)} \xi_{n}^{(0)}(y) d y \\
& =\frac{\sqrt{\sin \left(\frac{\pi(\eta+2 x)}{\epsilon}\right)+2}}{2 \sqrt{2} \pi^{2} n^{2}\left(\epsilon^{2} n^{2}-4\right)^{2}}\left[128 \sin \left(\pi n x+\frac{\pi n}{2}\right)+16 \epsilon^{2} n^{2} \sin \left(\frac{1}{2} \pi n(2 x+1)\right)\left(\sin \left(\frac{\pi(\eta+2 x)}{\epsilon}\right)-4\right)\right. \\
& +8 \epsilon^{3} n^{3}\left(-(-1)^{n}(2 x+1) \cos \left(\frac{\pi(\eta+1)}{\epsilon}\right)+\cos \left(\frac{\pi(2 \epsilon n x+\epsilon n-2 \eta-4 x)}{2 \epsilon}\right)\right. \\
& \left.+\cos \left(\frac{\pi(2 \epsilon n x+\epsilon n+2 \eta+4 x)}{2 \epsilon}\right)+(2 x-1) \cos \left(\frac{\pi-\pi \eta}{\epsilon}\right)\right) \\
& \left.+4 \epsilon^{4} n^{4} \sin \left(\pi n x+\frac{\pi n}{2}\right)\left(\sin \left(\frac{\pi(\eta+2 x)}{\epsilon}\right)+2\right)\right]
\end{aligned}
$$

To see if $\xi_{n}^{(1)}(x)$ are good approximations we estimate the mean square deviation using these states; if we let $\epsilon \rightarrow 0^{+}$keeping $n$ fixed we have

$$
\Delta_{n}^{(1)} \approx \frac{\sqrt{7}}{64} \pi^{2} \epsilon^{2} n^{4}+O\left(\epsilon^{3}\right)
$$

Therefore $\xi_{n}^{(1)}(x)$ are good approximations if $\Delta_{n}^{(1)} \ll 1$, implying $n \ll \frac{2 \sqrt{\frac{2}{\pi}}}{\sqrt[8]{7} \sqrt{\epsilon}} \approx \frac{1.25}{\sqrt{\epsilon}}$. Working in this limit, we may estimate the eigenvalues of the lowest part of the spectrum evaluating the 
expectation value of $\hat{O}$ :

$$
\begin{aligned}
E_{n} & \approx\langle\hat{O}\rangle_{\xi_{n}^{(1)}}=\frac{\int \xi_{n}^{(1)}(x) \hat{O} \xi_{n}^{(1)}(x) d x}{\int\left[\xi_{n}^{(1)}(x)\right]^{2} d x} \\
& \approx \frac{\pi^{2} n^{2}}{2}-\frac{1}{64} \pi^{2} \epsilon^{2} n^{4}+\frac{1}{4} \pi \epsilon^{3} n^{4} \sin \left(\frac{\pi}{\epsilon}\right) \sin \left(\frac{\pi \eta}{\epsilon}\right)+O\left[\epsilon^{4}\right]
\end{aligned}
$$

For $\eta=1$ this result reproduces the estimate obtained in refs. [16, 17].

\subsubsection{Neumann-Dirichlet and Dirichlet-Neumann boundary conditions}

Working in analogy to what we have done for the case of Dirichlet boundary conditions in the previous chapter, we use the ansatz

$$
\xi^{(0)}(x)=\sqrt{\Sigma(x)} \psi_{1}^{(N D)}(x)
$$

where

$$
\psi_{n}^{(N D)}(x) \equiv \sqrt{2} \sin \left(\frac{1}{4} \pi(2 n-1)(2 x+3)\right)
$$

are the Dirichlet eigenfunctions of the negative 1D Laplacian on $x \in(-1 / 2,1 / 2)$.

We apply Theorem 1 and after two iterations the expressions for the eigenvalue of the fundamental mode has converged to order $\epsilon$, for $\epsilon \rightarrow 0$ :

$$
E_{0}^{(N D)} \approx \frac{\pi^{2}}{8}-\frac{1}{16} \pi \epsilon \cos \left(\frac{\pi(1-\eta)}{\epsilon}\right)+O\left(\epsilon^{2}\right)
$$

Notice that the convergence in this case is slower than in the case of Dirichlet boundary conditions.

Observing that under the changes $\epsilon \rightarrow-\epsilon$ and $\eta \rightarrow-\eta$, we have that $\Sigma(x) \rightarrow \Sigma(-x)$, we are able to obtain the energy of the lowest mode for Dirichlet-Neumann boundary conditions for free:

$$
E_{0}^{(D N)} \approx \frac{\pi^{2}}{8}+\frac{1}{16} \pi \epsilon \cos \left(\frac{\pi(\eta+1)}{\epsilon}\right)+O\left(\epsilon^{2}\right)
$$

In Fig 7 we plot eq. (78) for three different values of $\eta$ (the curves for $\epsilon<0$ may be viewed as the asymptotic expression for $E_{0}^{(D N)}$ with phase $-\eta$ ). As for the case of Dirichlet boundary conditions we calculate the mean square deviations after one and two iterations, which read:

$$
\begin{aligned}
& \Delta^{(1)}=\left|\epsilon \cos \left(\frac{\pi(\eta-1)}{\epsilon}\right)\right| \frac{\pi}{64} \sqrt{\frac{1}{6}\left(\pi^{4}-96\right)}+O\left(\epsilon^{2}\right) \\
& \Delta^{(2)}=\left|\epsilon \cos \left(\frac{\pi(\eta-1)}{\epsilon}\right)\right| \frac{\pi}{768} \sqrt{\frac{17 \pi^{8}}{70}-2304}+O\left(\epsilon^{2}\right)
\end{aligned}
$$

For a general state, we use the initial ansatz $\xi_{n}^{(0)}(x)=\sqrt{\Sigma(x)} \psi_{n}^{(N D)}(x)$ and obtain the approximate expression for the $n^{\text {th }}$ eigenvalue after one iteration

$$
E_{n}^{(N D)} \approx \frac{\pi^{2}}{8}(1-2 n)^{2}-\frac{\pi}{16} \epsilon(1-2 n)^{2} \cos \left(\frac{\pi}{\epsilon}-\frac{\pi \eta}{\epsilon}\right)+O\left(\epsilon^{2}\right)
$$




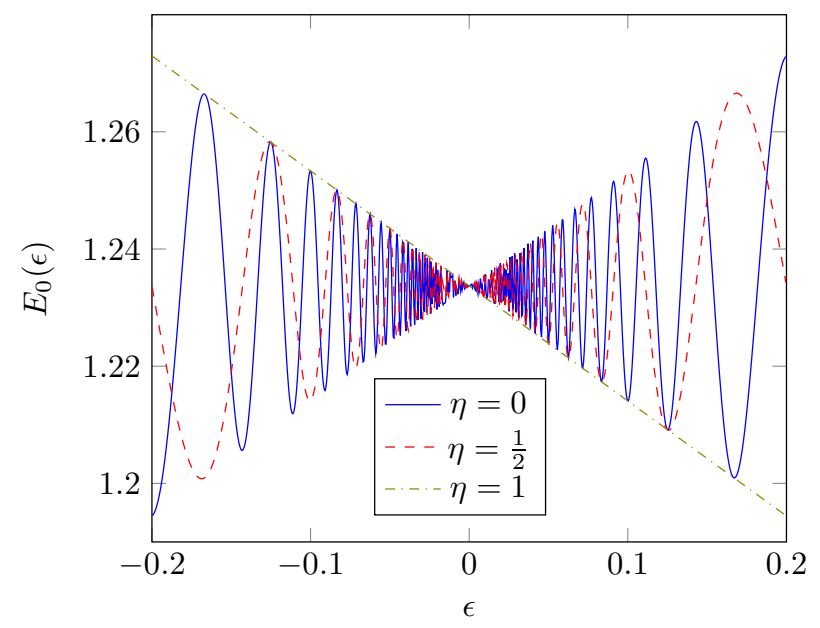

Figure 7: Eq.78 for three different values of $\eta$.

The corresponding mean square deviation in this case is

$$
\Delta_{n}^{(1)}=\frac{\pi(1-2 n)^{2}}{64 \sqrt{6}} \sqrt{\left(\pi^{4}(1-2 n)^{4}-96\right)}\left|\epsilon \cos \left(\frac{\pi(\eta-1)}{\epsilon}\right)\right|+O\left(\epsilon^{2}\right)
$$

which therefore requires $n \ll \epsilon^{-1 / 4}$.

\subsubsection{Neumann boundary conditions}

This case is slightly more complicated since the spectrum contains a zero mode. The implementation of the iterative method is done following Theorem 2 and using an initial ansatz orthogonal to the zero mode; we use the ansatz

$$
\begin{aligned}
\xi^{(0)}(x) & =\sqrt{\Sigma(x)} \psi_{1,2}^{(N N)}(x)-\frac{\int_{-a / 2}^{a / 2} \psi_{1,2}^{(N N)}(x) \Sigma(x) d x}{\int_{-a / 2}^{a / 2} \Sigma(x) d x} \sqrt{\Sigma(x)} \\
& =\sqrt{2} \sqrt{2+\sin \left(\frac{\pi(\eta+2 x)}{\epsilon}\right)}\left(\sin (\pi x)-\frac{4 \epsilon \cos \left(\frac{\pi}{\epsilon}\right) \cos \left(\frac{\pi \eta}{\epsilon}\right)}{\left(\epsilon^{2}-4\right)\left(\epsilon \sin \left(\frac{\pi}{\epsilon}\right) \sin \left(\frac{\pi \eta}{\epsilon}\right)+2 \pi\right)}\right)
\end{aligned}
$$

where

$$
\psi_{n, u}^{(N N)}(x)=\left\{\begin{array}{cc}
\sqrt{\frac{1}{a}} & , \quad n=0, u=1 \\
\sqrt{\frac{2}{a}} \cos \frac{2 n \pi x}{a} & , \quad n>0, u=1 \\
\sqrt{\frac{2}{a}} \sin \frac{(2 n-1) \pi x}{a} & , \quad n>0, u=2
\end{array}\right.
$$




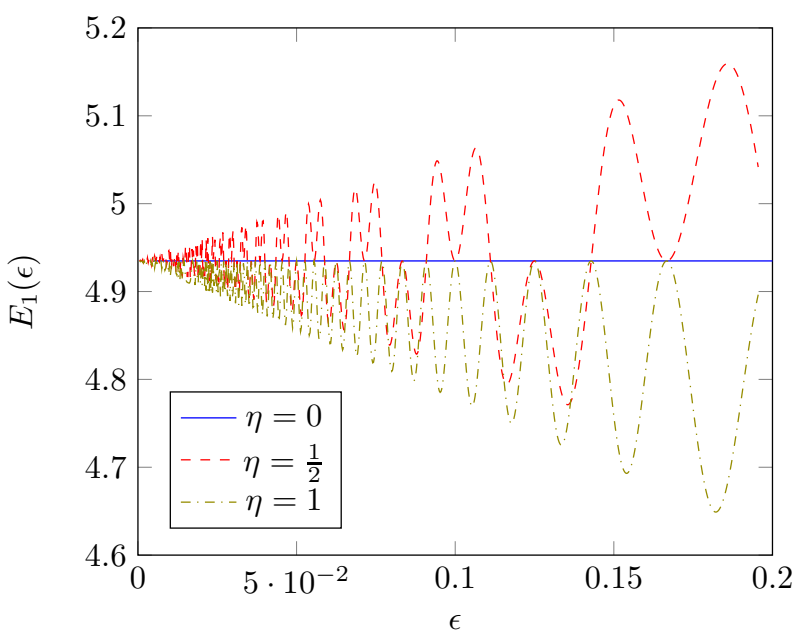

Figure 8: Eq.86 for three different values of $\eta$.

are the eigenfunction of the negative Laplacian obeying Neumann boundary conditions.

After one iteration we find

$$
E_{0}^{(N N)} \approx \frac{\pi^{2}}{2}-\frac{\pi \epsilon}{2} \sin \left(\frac{\pi}{\epsilon}\right) \sin \left(\frac{\pi \eta}{\epsilon}\right)+O\left(\epsilon^{2}\right)
$$

Notice that for $\eta=1$ this result agrees with the approximate expression obtained in Eq.(53) of Ref. [20].

The mean square deviation calculated after one iteration is

$$
\begin{aligned}
\Delta^{(1)} & \approx \frac{\pi|\epsilon|}{48 \sqrt{5}}\left[\left(-360 \cos \left(\frac{2 \pi(\eta-1)}{\epsilon}\right)+\left(720-7 \pi^{4}\right) \cos \left(\frac{2 \pi \eta}{\epsilon}\right)\right.\right. \\
& \left.\left.+\cos \left(\frac{2 \pi}{\epsilon}\right)\left(8 \pi^{4} \cos \left(\frac{2 \pi \eta}{\epsilon}\right)-7 \pi^{4}+720\right)+8\left(-45 \cos \left(\frac{2 \pi(\eta+1)}{\epsilon}\right)+\pi^{4}-90\right)\right)\right]^{1 / 2} \\
& +O\left(\epsilon^{2}\right)
\end{aligned}
$$

\subsubsection{Periodic boundary conditions}

In this case we consider the ansatz

$$
\begin{aligned}
\xi^{(0)}(x) & =\sqrt{\Sigma(x)}\left(\cos \phi \psi_{1,1}^{(P P)}(x)+\sin \phi \psi_{1,2}^{(P P)}(x)\right) \\
& -\frac{\int_{-a / 2}^{a / 2}\left(\cos \phi \psi_{1,1}^{(P)}(x)+\sin \phi \psi_{1,2}^{(P P)}(x)\right) \Sigma(x) d x}{\int_{-a / 2}^{a / 2} \Sigma(x) d x} \sqrt{\Sigma(x)}
\end{aligned}
$$


where

$$
\psi_{n, u}^{(P P)}(x)=\left\{\begin{array}{cc}
\sqrt{\frac{1}{a}} & , \quad n=0, u=1 \\
\sqrt{\frac{2}{a}} \cos \frac{2 n \pi x}{a} & , \quad n>0, u=1 \\
\sqrt{\frac{2}{a}} \sin \frac{2 n \pi x}{a} & , \quad n>0, u=2
\end{array}\right.
$$

are the eigenfunctions of the 1D negative Laplacian obeying periodic boundary conditions. Remember that these eigenfunctions are twice degenerate for $n>0$ : the ansatz is expressed in terms of the lowest two degenerate states.

The explicit form of this ansatz is

$$
\begin{aligned}
\xi^{(0)}(x) & =\sqrt{2} \sqrt{2+\sin \left(\frac{\pi(\eta+2 x)}{\epsilon}\right)}(\sin (\phi) \sin (2 \pi x)+\cos (\phi) \cos (2 \pi x) \\
& \left.-\frac{\epsilon \sin \left(\frac{\pi}{\epsilon}\right)\left(\epsilon \sin (\phi) \cos \left(\frac{\pi \eta}{\epsilon}\right)+\cos (\phi) \sin \left(\frac{\pi \eta}{\epsilon}\right)\right)}{\left(\epsilon^{2}-1\right)\left(\epsilon \sin \left(\frac{\pi}{\epsilon}\right) \sin \left(\frac{\pi \eta}{\epsilon}\right)+2 \pi\right)}\right)
\end{aligned}
$$

Applying Theorem 2 after one iteration, for $\epsilon \rightarrow 0$, the Rayleigh quotient provides the estimate for the lowest non-trivial eigenvalues

$$
E_{1,2}^{(P P)}=2 \pi^{2}-2 \pi \epsilon \sin \left(\frac{\pi}{\epsilon}\right) \cos ^{2}(\phi) \sin \left(\frac{\pi \eta}{\epsilon}\right)+O\left(\epsilon^{2}\right)
$$

The mean square deviation obtained using $\xi^{(1)}(x)$, for $\epsilon \rightarrow 0$, behaves as

$$
\Delta^{(1)}=\frac{1}{3} \sqrt{\frac{2}{5}} \pi\left|\epsilon \cos (\phi) \sin \left(\frac{\pi}{\epsilon}\right) \sin \left(\frac{\pi \eta}{\epsilon}\right)\right| \sqrt{\pi^{4}-45 \cos (2 \phi)-45}+O\left(\epsilon^{2}\right)
$$

In Fig. 9 , we plot the quantity $\tilde{\Delta} \equiv|\cos (\phi)| \sqrt{\pi^{4}-45 \cos (2 \phi)-45}$ as a function of the parameter $\phi$ which controls the mixing of the degenerate states in the initial ansatz. As we see from the figure, the mean square deviation goes to zero to order $\epsilon$ at $\phi=\pi / 2$, while it has a local minimum at $\phi=0$. This suggests that the two lowest degenerate states of homogeneous problem split at finite $\epsilon$ into a state with constant energy (to order $\epsilon$ ) and a state with oscillating energy. In Fig.10 we display the asymptotic formulas (89) for $\eta=1$ (corresponding to $\phi=0$ and $\phi=\pi / 2$ ) and compare them with the numerical results obtained with the Rayleigh-Ritz method using a set of 101 states. The agreement between the analytical and the numerical results is evident. A similar agreement was also observed for the cases for $\eta=0$ and $\eta=1 / 2$, although we do not include any figure for these cases.

\section{Conclusions}

We have devised an iterative method which allows one to obtain arbitrarily precise solutions to the lowest eigenvalues and eigenfunctions of the Helmholtz equation for an heterogeneous medium in $d$ dimensions, obeying different boundary conditions. The method requires the knowledge of the Green's function for the corresponding homogeneous problem and therefore it is limited to certain class of domains (in two dimensions, however, one can apply it to arbitrary simply connected domains, which can be conformally mapped to a circle or to a square, for 


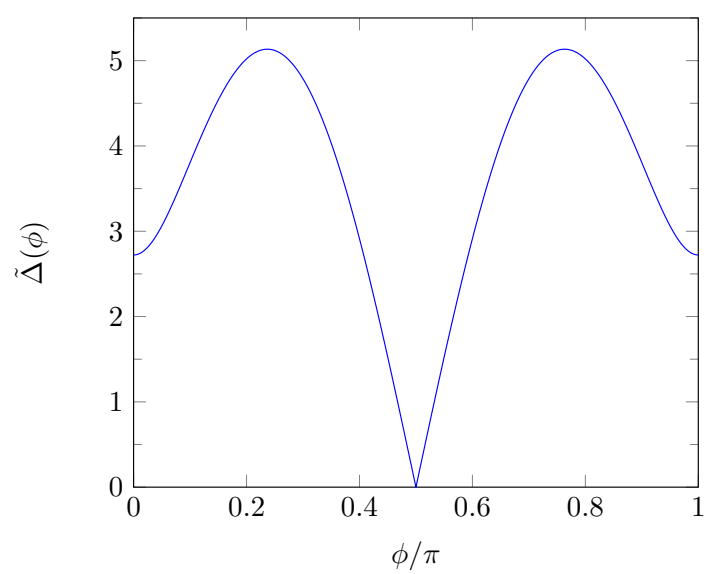

Figure 9: Reduced mean square deviation $\tilde{\Delta}$ as a function of $\phi$.

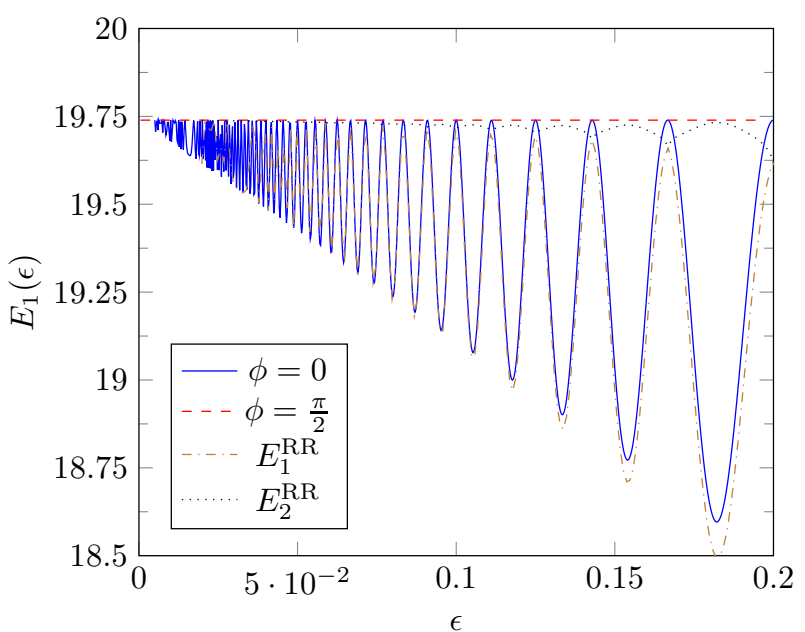

Figure 10: Asymptotic behavior of $E_{1,2}(\epsilon)$ for $\eta=1$ obtained using Theorem 2 and numerical results obtained with the Rayleigh-Ritz method. 
which explicit expressions for the homogeneous Green's functions are available). A sequence of approximations which converges to the targeted solution is obtained applying iteratively the inverse operator of Eq. (A.2) to an initial arbitrary ansatz, with a finite overlap with the targeted state. Some applications in one and two dimensions are used to illustrate the method. In particular we have obtained the asymptotic behaviour of the lowest part of the spectrum of a string with a rapidly oscillating density when the size of the micro-structure goes to zero, for Dirichlet, Dirichlet-Neumann, Neumann and periodic boundary conditions.

\section{Appendix A. Inverse operator}

In this paper we have considered the operator

$$
\hat{O}=\frac{1}{\sqrt{\Sigma(\mathbf{x})}}(-\Delta) \frac{1}{\sqrt{\Sigma(\mathbf{x})}}
$$

We are interested in the spectrum of this operator on a $d$-dimensional region $\Omega_{d}$, where its eigenfunctions obey appropriate boundary conditions; depending on the boundary conditions the spectrum may contain a zero mode.

Let us first consider the case in which a zero mode is not present and let $G(\mathbf{x}, \mathbf{y})$ be the Green's function of the negative Laplacian on $\Omega_{d}$ corresponding to the specific boundary conditions. We assume that $G(\mathbf{x}, \mathbf{y})$ is known exactly (which in general is not true). Under these assumptions it is straightforward to check that the operator

$$
\hat{P} f=\sqrt{\Sigma(\mathbf{x})} \int d^{d} y G(\mathbf{x}, \mathbf{y}) \sqrt{\Sigma(\mathbf{y})} f(\mathbf{y})
$$

is the inverse of $\hat{O}$. As a matter of fact

$$
\begin{aligned}
\hat{O} \hat{P} f & =\frac{1}{\sqrt{\Sigma(\mathbf{x})}}(-\Delta) \int d^{d} y G(\mathbf{x}, \mathbf{y}) \sqrt{\Sigma(\mathbf{y})} f(\mathbf{y}) \\
& =\frac{1}{\sqrt{\Sigma(\mathbf{x})}} \int d^{d} y \delta(\mathbf{x}-\mathbf{y}) \sqrt{\Sigma(\mathbf{y})} f(\mathbf{y})=f(\mathbf{x})
\end{aligned}
$$

The case in which a zero mode is present is more delicate. In this case we assume that $G(\mathbf{x}, \mathbf{y})$ is the regularized Green's function, which does not contain the divergent contribution of the zero mode. In this case one has

$$
(-\Delta) G(\mathbf{x}, \mathbf{y})=\delta(\mathbf{x}-\mathbf{y})-\frac{1}{V_{\Omega}}
$$

and

$$
\begin{aligned}
\hat{O} \hat{P} f & =\frac{1}{\sqrt{\Sigma(\mathbf{x}})}(-\Delta) \int d^{d} y G(\mathbf{x}, \mathbf{y}) \sqrt{\Sigma(\mathbf{y})} f(\mathbf{y}) \\
& =\frac{1}{\sqrt{\Sigma(\mathbf{x})}} \int d^{d} y\left[\delta(\mathbf{x}-\mathbf{y})-\frac{1}{V_{\Omega}}\right] \sqrt{\Sigma(\mathbf{y})} f(\mathbf{y}) \\
& =f(\mathbf{x})-\frac{1}{V_{\Omega}} \frac{1}{\sqrt{\Sigma(\mathbf{x})}} \int d^{d} y \sqrt{\Sigma(\mathbf{y})} f(\mathbf{y})
\end{aligned}
$$

Therefore we see that, for a generic $f$ the operator $\hat{P}$ is not the inverse of $\hat{O}$ : however, if $f(\mathbf{x})$ is orthogonal to the zero mode, whose eigenfunction is precisely $\sqrt{\Sigma(\mathbf{x})}$, then it follows that $\int d^{d} y \sqrt{\Sigma(\mathbf{y})} f(\mathbf{y})=0$ and $\hat{P}$ behaves as $\hat{O}^{-1}$. 


\section{Appendix B. Matrix representation}

In some situations it may be convenient to use the explicit representation of the Green's function for the homogeneous problem. For the case where a zero mode is not present the Green's function reads

$$
G\left(x_{1}, \ldots, x_{d} ; y_{1}, \ldots, y_{d}\right)=\sum_{n} \frac{\phi_{n}\left(x_{1}, \ldots, x_{d}\right) \phi_{n}\left(y_{1}, \ldots, y_{d}\right)}{\epsilon_{n}}
$$

where $n$ is the set of all quantum numbers which specify an eigenstate of the homogeneous problem.

The iterations obtained applying Theorem 1 to the initial ansatz $\Xi^{(1)}=\sqrt{\Sigma} \phi_{n_{0}}$ can then be obtained in the form of multiple series whose summands involve the matrix elements of the density:

$$
\begin{aligned}
\Xi^{(1)}\left(x_{1}, \ldots, x_{d}\right) & =\sqrt{\Sigma\left(x_{1}, \ldots, x_{d}\right)} \sum_{n_{1}} \frac{\left\langle n_{1}|\Sigma| 1\right\rangle}{\epsilon_{n_{1}}} \phi_{n_{1}}\left(x_{1}, \ldots, x_{d}\right) \\
\Xi^{(2)}\left(x_{1}, \ldots, x_{d}\right) & =\sqrt{\Sigma\left(x_{1}, \ldots, x_{d}\right)} \sum_{n_{1}, n_{2}} \frac{\left\langle n_{1}|\Sigma| n_{2}\right\rangle\left\langle n_{2}|\Sigma| 1\right\rangle}{\epsilon_{n_{1}} \epsilon_{n_{2}}} \phi_{n_{1}}\left(x_{1}, \ldots, x_{d}\right) \\
& \ldots \\
\Xi^{(p)}\left(x_{1}, \ldots, x_{d}\right) & =\sqrt{\Sigma\left(x_{1}, \ldots, x_{d}\right)} \sum_{n_{1}, n_{2}, \ldots, n_{p}} \frac{\left\langle n_{1}|\Sigma| n_{2}\right\rangle \ldots\left\langle n_{p}|\Sigma| 1\right\rangle}{\epsilon_{n_{1}} \ldots \epsilon_{n_{p}}} \phi_{n_{1}}\left(x_{1}, \ldots, x_{d}\right)
\end{aligned}
$$

These expressions can be used to cast the integrals $\int_{\Omega_{d}} d^{d} x\left[\Xi^{(p)}\right]^{2}$ and $\int_{\Omega_{d}} d^{d} x\left[\Xi^{(p)} \Xi^{(p-1)}\right]$ in the form of infinite series. This implementation of Theorem 1 corresponds essentially to the approach of Ref. [22].

Let us apply the operator $\hat{O}=\frac{1}{\sqrt{\Sigma}}(-\Delta) \frac{1}{\sqrt{\Sigma}}$ to the function obtained after $p$ iterations:

$$
\hat{O} \Xi^{(p)}\left(x_{1}, \ldots, x_{d}\right)=\frac{1}{\sqrt{\Sigma(x)}} \sum_{n_{1}, n_{2}, \ldots, n_{p}} \frac{\left\langle n_{1}|\Sigma| n_{2}\right\rangle \ldots\left\langle n_{p}|\Sigma| 1\right\rangle}{\epsilon_{n_{2}} \ldots \epsilon_{n_{p}}} \phi_{n_{1}}\left(x_{1}, \ldots, x_{d}\right)
$$

where we have used the fact that $\phi_{n_{1}}\left(x_{1}, \ldots, x_{d}\right)$ is an eigenfunction of the negative Laplacian. We now use the completeness of the basis of the eigenfunctions of the Laplacian to write

$$
\sum_{n_{1}}\left\langle n_{1}|\Sigma| n_{2}\right\rangle \phi_{n_{1}}\left(x_{1}, \ldots, x_{d}\right)=\Sigma(x) \phi_{n_{2}}\left(x_{1}, \ldots, x_{d}\right)
$$

and therefore

$$
\begin{aligned}
\hat{O} \Xi^{(p)}\left(x_{1}, \ldots, x_{d}\right) & =\sqrt{\Sigma(x)} \sum_{n_{2}, \ldots, n_{p}} \frac{\left\langle n_{2}|\Sigma| n_{3}\right\rangle \ldots\left\langle n_{p}|\Sigma| 1\right\rangle}{\epsilon_{n_{2}} \ldots \epsilon_{n_{p}}} \phi_{n_{2}}\left(x_{1}, \ldots, x_{d}\right) \\
& =\Xi^{(p-1)}\left(x_{1}, \ldots, x_{d}\right)
\end{aligned}
$$

which confirms that the operator in Eq. A.2 is indeed the inverse operator of $\hat{O}$.

It useful to discuss also the case in which the zero mode is present. In this case we need to apply Theorem 2 starting from an ansatz

$$
\tilde{\Xi}^{(0)}(\mathbf{x})={ }_{28}^{\sqrt{\Sigma(\mathbf{x})}} \phi_{\mathbf{n}_{0}}(\mathbf{x})
$$


which is then orthogonalized with respect to the zero mode:

$$
\Xi^{(0)}(\mathbf{x})=\sqrt{\Sigma(\mathbf{x})}\left[\phi_{\mathbf{n}_{0}}(\mathbf{x})-\frac{1}{\sqrt{\Omega_{d}}} \frac{\left\langle\mathbf{n}_{\mathbf{0}}|\Sigma| 0\right\rangle}{\langle 0|\Sigma| 0\rangle}\right]
$$

After one iteration we have

$$
\tilde{\Xi}^{(1)}(\mathbf{x})=\sqrt{\Sigma(\mathbf{x})} \sum_{n_{1}}^{\prime} \frac{\phi_{\mathbf{n}_{1}}(\mathbf{x})}{\epsilon_{\mathbf{n}_{1}}}\left[\left\langle\mathbf{n}_{1}|\Sigma| \mathbf{n}_{0}\right\rangle-\frac{\left\langle\mathbf{n}_{1}|\Sigma| 0\right\rangle\left\langle\mathbf{n}_{0}|\Sigma| 0\right\rangle}{\langle 0|\Sigma| 0\rangle}\right]
$$

and

$$
\Xi^{(1)}(\mathbf{x})=\sqrt{\Sigma(\mathbf{x})} \sum_{n_{1}}^{\prime} \frac{1}{\epsilon_{\mathbf{n}_{1}}}\left(\phi_{\mathbf{n}_{1}}(\mathbf{x})-\frac{1}{\sqrt{\Omega_{d}}} \frac{\left\langle\mathbf{n}_{1}|\Sigma| 0\right\rangle}{\langle 0|\Sigma| 0\rangle}\right) \cdot\left(\left\langle\mathbf{n}_{1}|\Sigma| \mathbf{n}_{0}\right\rangle-\frac{\left\langle\mathbf{n}_{1}|\Sigma| 0\right\rangle\left\langle\mathbf{n}_{0}|\Sigma| 0\right\rangle}{\langle 0|\Sigma| 0\rangle}\right) \mathrm{B} .1
$$

After two iterations we have

$$
\begin{aligned}
\tilde{\Xi}^{(2)}(\mathbf{x})= & \sqrt{\Sigma(\mathbf{x})} \sum_{n_{1}, n_{2}}^{\prime} \frac{\phi_{\mathbf{n}_{1}}(\mathbf{x})}{\epsilon_{\mathbf{n}_{1}} \epsilon_{\mathbf{n}_{2}}}\left(\left\langle\mathbf{n}_{1}|\Sigma| \mathbf{n}_{2}\right\rangle-\frac{\left\langle\mathbf{n}_{1}|\Sigma| 0\right\rangle\left\langle\mathbf{n}_{2}|\Sigma| 0\right\rangle}{\langle 0|\Sigma| 0\rangle}\right) \\
\cdot & \left(\left\langle\mathbf{n}_{2}|\Sigma| \mathbf{n}_{0}\right\rangle-\frac{\left\langle\mathbf{n}_{2}|\Sigma| 0\right\rangle\left\langle\mathbf{n}_{0}|\Sigma| 0\right\rangle}{\langle 0|\Sigma| 0\rangle}\right)
\end{aligned}
$$

and

$$
\begin{aligned}
\Xi^{(2)}(\mathbf{x})= & \sqrt{\Sigma(\mathbf{x})} \sum_{n_{1}, n_{2}}^{\prime} \frac{1}{\epsilon_{\mathbf{n}_{1}} \epsilon_{\mathbf{n}_{2}}}\left(\phi_{\mathbf{n}_{1}}(\mathbf{x})-\frac{1}{\sqrt{\Omega_{d}}} \frac{\left\langle\mathbf{n}_{1}|\Sigma| 0\right\rangle}{\langle 0|\Sigma| 0\rangle}\right)\left(\left\langle\mathbf{n}_{1}|\Sigma| \mathbf{n}_{2}\right\rangle-\frac{\left\langle\mathbf{n}_{1}|\Sigma| 0\right\rangle\left\langle\mathbf{n}_{2}|\Sigma| 0\right\rangle}{\langle 0|\Sigma| 0\rangle}\right) \\
\cdot & \left(\left\langle\mathbf{n}_{2}|\Sigma| \mathbf{n}_{0}\right\rangle-\frac{\left\langle\mathbf{n}_{2}|\Sigma| 0\right\rangle\left\langle\mathbf{n}_{0}|\Sigma| 0\right\rangle}{\langle 0|\Sigma| 0\rangle}\right)
\end{aligned}
$$

Similarly, after $p$ iterations, one has

$$
\begin{aligned}
\tilde{\Xi}^{(p)}(\mathbf{x})= & \sqrt{\Sigma(\mathbf{x})} \sum_{n_{1}, \ldots, n_{p}}^{\prime} \frac{\phi_{\mathbf{n}_{1}}(\mathbf{x})}{\epsilon_{\mathbf{n}_{1}} \ldots \epsilon_{\mathbf{n}_{p}}}\left(\left\langle\mathbf{n}_{1}|\Sigma| \mathbf{n}_{2}\right\rangle-\frac{\left\langle\mathbf{n}_{1}|\Sigma| 0\right\rangle\left\langle\mathbf{n}_{2}|\Sigma| 0\right\rangle}{\langle 0|\Sigma| 0\rangle}\right) \ldots \\
& \cdot\left(\left\langle\mathbf{n}_{p}|\Sigma| \mathbf{n}_{0}\right\rangle-\frac{\left\langle\mathbf{n}_{p}|\Sigma| 0\right\rangle\left\langle\mathbf{n}_{0}|\Sigma| 0\right\rangle}{\langle 0|\Sigma| 0\rangle}\right)
\end{aligned}
$$

and

$$
\begin{aligned}
\Xi^{(p)}(\mathbf{x})= & \sqrt{\Sigma(\mathbf{x})} \sum_{n_{1}, \ldots, n_{p}}^{\prime} \frac{1}{\epsilon_{\mathbf{n}_{1}} \ldots \epsilon_{\mathbf{n}_{p}}}\left(\phi_{\mathbf{n}_{1}}(\mathbf{x})-\frac{1}{\sqrt{\Omega_{d}}} \frac{\left\langle\mathbf{n}_{1}|\Sigma| 0\right\rangle}{\langle 0|\Sigma| 0\rangle}\right)\left(\left\langle\mathbf{n}_{1}|\Sigma| \mathbf{n}_{2}\right\rangle-\frac{\left\langle\mathbf{n}_{1}|\Sigma| 0\right\rangle\left\langle\mathbf{n}_{2}|\Sigma| 0\right\rangle}{\langle 0|\Sigma| 0\rangle}\right) \ldots \\
& \cdot\left(\left\langle\mathbf{n}_{p}|\Sigma| \mathbf{n}_{0}\right\rangle-\frac{\left\langle\mathbf{n}_{p}|\Sigma| 0\right\rangle\left\langle\mathbf{n}_{0}|\Sigma| 0\right\rangle}{\langle 0|\Sigma| 0\rangle}\right)
\end{aligned}
$$

Let us now apply the operator $\hat{O}$ to $\Xi^{(p)}(\mathbf{x})$, using the properties discussed for the previous case:

$$
\begin{aligned}
\hat{O} \Xi^{(p)}(\mathbf{x})= & \frac{1}{\sqrt{\Sigma(\mathbf{x})}} \sum_{n_{1}, \ldots, n_{p}}^{\prime} \frac{1}{\epsilon_{\mathbf{n}_{2}} \ldots \epsilon_{\mathbf{n}_{p}}} \phi_{\mathbf{n}_{1}}(\mathbf{x})\left(\left\langle\mathbf{n}_{1}|\Sigma| \mathbf{n}_{2}\right\rangle-\frac{\left\langle\mathbf{n}_{1}|\Sigma| 0\right\rangle\left\langle\mathbf{n}_{2}|\Sigma| 0\right\rangle}{\langle 0|\Sigma| 0\rangle}\right) \ldots \\
& \cdot\left(\left\langle\mathbf{n}_{p}|\Sigma| \mathbf{n}_{0}\right\rangle-\frac{\left\langle\mathbf{n}_{p}|\Sigma| 0\right\rangle\left\langle\mathbf{n}_{0}|\Sigma| 0\right\rangle}{\langle 0|\Sigma| 0\rangle}\right) \\
29 &
\end{aligned}
$$


Now, using the completeness of the basis, one has

$$
\sum_{n_{1}, \ldots, n_{p}}^{\prime} \phi_{\mathbf{n}_{1}}(\mathbf{x})\left(\left\langle\mathbf{n}_{1}|\Sigma| \mathbf{n}_{2}\right\rangle-\frac{\left\langle\mathbf{n}_{1}|\Sigma| 0\right\rangle\left\langle\mathbf{n}_{2}|\Sigma| 0\right\rangle}{\langle 0|\Sigma| 0\rangle}\right)=\Sigma(\mathbf{x})\left(\phi_{\mathbf{n}_{2}}(\mathbf{x})-\frac{1}{\sqrt{\Omega_{d}}} \frac{\left\langle\mathbf{n}_{2}|\Sigma| 0\right\rangle}{\langle 0|\Sigma| 0\rangle}\right)
$$

and therefore

$$
\begin{aligned}
\hat{O} \Xi^{(p)}(\mathbf{x})= & \sqrt{\Sigma(\mathbf{x})} \sum_{n_{2}, \ldots, n_{p}}^{\prime} \frac{1}{\epsilon_{\mathbf{n}_{2}} \ldots \epsilon_{\mathbf{n}_{p}}}\left(\phi_{\mathbf{n}_{2}}(\mathbf{x})-\frac{1}{\sqrt{\Omega_{d}}} \frac{\left\langle\mathbf{n}_{2}|\Sigma| 0\right\rangle}{\langle 0|\Sigma| 0\rangle}\right)\left(\left\langle\mathbf{n}_{2}|\Sigma| \mathbf{n}_{3}\right\rangle-\frac{\left\langle\mathbf{n}_{2}|\Sigma| 0\right\rangle\left\langle\mathbf{n}_{3}|\Sigma| 0\right\rangle}{\langle 0|\Sigma| 0\rangle}\right) \ldots \\
& \cdot\left(\left\langle\mathbf{n}_{p}|\Sigma| \mathbf{n}_{0}\right\rangle-\frac{\left\langle\mathbf{n}_{p}|\Sigma| 0\right\rangle\left\langle\mathbf{n}_{0}|\Sigma| 0\right\rangle}{\langle 0|\Sigma| 0\rangle}\right)=\Xi^{(p-1)}(\mathbf{x})
\end{aligned}
$$

which confirms the conclusion in the previous appendix that operator in Eq. (A.2) is indeed the inverse operator of $\hat{O}$ for the class of functions that we are considering.

\section{Acknowledgements}

This research was supported by the Sistema Nacional de Investigadores (México). The plots contained in this paper have been produced using TikZ [34].

\section{References}

[1] J.A. Masad, Journal of Sound and Vibration 195, 674-678 (1996)

[2] C.Y. Wang, Journal of Sound and Vibration 210, 555-558 (1998)

[3] C.O. Horgan and A.M. Chan, Journal of Sound and Vibration 225, 503-513 (1999)

[4] S.H. Ho and C.K.Chen, Journal of Sound and Vibration 233, 547-555 (2000)

[5] S.W. Kang and J.M. Lee, Journal of Sound and Vibration 251, 505-517 (2002)

[6] S.D. M. Adams, R.V. Craster and S. Guenneau, Proc. R. Soc. A., , (2008)

[7] W.J. Parnell and I.D. Abrahams, Wave Motion 43, 474-498 (2006)

[8] V.P. Smyshlyaev, Mechanics of Materials 41, 434-447 (2009)

[9] Y. Capdeville and J.J. Marigo, Geophys. J. Int. 170, 823-838 (2007)

[10] Y. Capdeville, L. Guillot and J.J. Marigo, Geophys. J. Int. (2007)

[11] S. Nemat-Nasser and J.R. Willis, Phys. Rev. B 83, 104103 (2011)

[12] A. Srivastava and S. Nemat-Nasser, Proc. R. Soc. A, 269-287 (2012)

[13] E. Rohan, Wave motion 50, 1073-1089 (2013)

[14] J.D. Joannopoulos, P.R. Villeneuve and S. Fan, Nature 386, 143-149 (1997)

[15] G.P. Panasenko, Physics of Atomic Nuclei 71, 681-694 (2008)

[16] C. Castro and E. Zuazua, SIAM J. Appl. Math. 60, 1205-1233 (2000)

[17] P. Amore, Annals of Physics 326, 23152355 (2011)

[18] P. Amore, Annals of Physics 349, 253-267 (2014)

[19] P. Amore, Annals of Physics 338, 341360 (2013)

[20] P. Amore, Annals of Physics 336, 223-244 (2013)

[21] P. Amore, Annals of Physics 325, 2679-2696 (2010)

[22] P. Amore, J. Math. Phys. 51, 052105 (2010)

[23] P. Amore, J. Math. Phys. 53, 123519 (2012)

[24] M. Robnik, J.Phys. A 17, 1049 (1984)

[25] C. Lanczos, J. Res. Nat. Bur. Stand. 45, 255 (1950)

[26] W.A. Berger, H.G. Miller, K-G. Kreuzer and R. M. Dreizler, J. Phys. A 10, 1089-1095 (1977)

[27] E. Dagotto and A. Moreo, Phys. Rev. D 31, 865-870 (1985)

[28] E.R. Gagliano, E. Dagotto, A. Moreo and F.C. Alcaraz, Phys. Rev. B 34, 1677-1682 (1986)

[29] L.W. Wang and A. Zunger, J. Chem. Phys. 100, 2394 (1994)

[30] L.W. Wang and A. Zunger, Phys. Rev. B 53, 9579 (1996) 
[31] John David Jackson, Classical Electrodynamics, Third ed., Wiley, New York, 1998

[32] D. Cioranescu and P. Donato, An introduction to homogenization, Oxford University Press (1999)

[33] L. Tartar, The general theory of homogenization, Springer (2009)

[34] Till Tantau, The TikZ and PGF Packages, Manual for version 3.0.0, http://sourceforge.net/projects/pgf/. 2013-12-20 